\title{
PlanetEvidence: Planet or Noise?
}

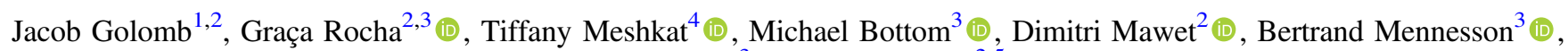 \\ Gautam Vasisht ${ }^{3}$, and Jason Wang ${ }^{2,5}$ (D) \\ ${ }^{1}$ University of Maryland, College Park, MD, 20742, USA; golombj@umd.edu \\ ${ }^{2}$ California Institute of Technology, MC 249-17, 1200 East California Boulevard, Pasadena, CA 91125, USA; graca@caltech.edu, graca.m.rocha@jpl.nasa.gov \\ ${ }^{3}$ Jet Propulsion Laboratory, California Institute of Technology, 4800 Oak Grove Drive, Pasadena, CA 91109, USA \\ ${ }^{4}$ Infrared Processing and Analysis Center, MC 100-22 or MC 314-6, 1200 East California Boulevard, Pasadena, CA 91125, USA \\ Received 2019 November 27; revised 2021 June 1; accepted 2021 June 21; published 2021 December 9
}

\begin{abstract}
The work presented here attempts at answering the following question: how do we decide when a given detection is a planet or just residual noise in exoplanet direct imaging data? To this end we implement a metric meant to replace the empirical frequentist-based thresholds for detection. Our method, implemented within a Bayesian framework, introduces an "evidence-based" approach to help decide whether a given detection is a true planet or just noise. We apply this metric jointly with a postprocessing technique and Karhunen-Loeve Image Processing (KLIP), which models and subtracts the stellar PSF from the image. As a proof of concept we implemented a new routine named PlanetEvidence that integrates the nested sampling technique (Multinest) with the KLIP algorithm. This is a first step to recast such a postprocessing method into a fully Bayesian perspective. We test our approach on real direct imaging data, specifically using GPI data of $\beta$ Pictoris $b$, and on synthetic data. We find that for the former the method strongly favors the presence of a planet (as expected) and recovers the true parameter posterior distributions. For the latter case our approach allows us to detect (true) dim sources invisible to the naked eye as real planets, rather than background noise, and set a new lower threshold for detection at $\sim 2.5 \sigma$ level. Further it allows us to quantify our confidence that a given detection is a real planet and not just residual noise.
\end{abstract}

Unified Astronomy Thesaurus concepts: Exoplanet detection methods (489); Direct imaging (387); Bayesian statistics (1900); Exoplanets (498)

\section{Introduction}

Detection and characterization of extrasolar planets has become a flourishing field in the last two decades or so. Several detection techniques have been applied: indirect, such as "radial-velocity" and "transit" detection, microlensing, and "direct imaging" techniques. The latter offers a unique way to study exoplanets in the context of their formation and evolution. While indirect techniques have resulted in the discovery of thousands of planets (mainly thanks to the Kepler mission), direct imaging has discovered only a handful of planets. On one hand the difficulty arises from the residual glare of starlight at small orbital separations where most planets are expected to reside. On the other hand fewer giant gaseous planets are expected at large angular separations (Nielsen et al. 2019; Vigan et al. 2020). One measure of the detectability of a planet is the "raw contrast" (hereafter referred to as "contrast"), defined as the ratio of the average starlight irradiance in a region of interest to the average irradiance of unblocked starlight in an equivalent aperture centered on the star. The problem resides in the resolution and contrast needed to confirm the presence of a planet in the image (Oppenheimer \& Hinkley 2009).

For example the Coronagraph Instrument (CGI) on the Wide-Field Infrared Survey Telescope will aim to reach raw contrasts of about 1e-9 to 1e-8 using state-of-the-art starlight suppression and wave-front control techniques in order to detect such planets. A further contrast improvement of at least a factor of two is expected at the postprocessing step.

In order to reach such deep sensitivity limits, new instruments and image processing techniques have been

\footnotetext{
51 Pegasi b Fellow.
}

developed. For example, coronagraphs are used to block light from the star, thus suppressing the flux from the point-spread function (PSF) of the star, while leaving the planet visible. Its optics allow us to reach smaller inner working angles, but are affected by the stellar speckles which can dominate the flux from the image plane (Bottom et al. 2017).

At the postprocessing stage there are a number of image processing techniques that aim at modeling and subtracting the stellar PSF to allow the planet to become detectable, in effect increasing the contrast achievable next to a bright star. In terms of characterization techniques, the state of the art is: (1) visual detection and negative fake companion injections (Lagrange et al. 2009); (2) forward modeling after the subtraction (Mugnier et al. 2009; Cantalloube et al. 2015; Pueyo 2016; Ruffio et al. 2017); and (3) statistical sampling in the subtracted image, e.g, pyKLIP-MCMC (Wang et al. 2016).

In this paper, we build on a class of techniques known as principal component analysis (PCA; Amara \& Quanz 2012), which model the temporal variation of the PSF by identifying the main linear components of the temporal variation and the related Karhunen-Loeve Image Processing (KLIP; Soummer et al. 2012), which uses the Karhunen-Loeve (KL) transform to model the PSF. It has been shown that PCA-based methods achieve greater sensitivity and that varying and optimizing the number of principal components is one of the best ways to enhance the planet signal (Amara \& Quanz 2012; Soummer et al. 2012).

While these methods enhance the detectability of faint astrophysical signals, they are susceptible to systematic biases due to mismodeling of the PSF, which may lead to over- and under-subtraction of stellar light inducing either (i) loss of planetary signals or (ii) strong starlight residuals. To tackle this issue, KLIP forward modeling (KLIP-FM; Pueyo 2016), 
perturbation-based KL image processing forward modeling has been developed. More recently, Ruffio et al. (2017, 2018) developed a forward model matched filter (FMMF), which uses the forward model provided by KLIP-FM as the template of the matched filter to boost the signal from the planet and hence enhance its detectability. ANDROMEDA and PACO also apply this correction.

PSF subtractions on both ground-based and space-based instruments have not yet achieved the contrast gain needed to detect planets with masses lower than 1 Jupiter mass at separations smaller than $0.1^{\prime \prime}$. Further study is required to enhance the detectability of planets faint signals hidden in the instrumental and background noise as well as to improve their characterization i.e., render an unbiased estimation of their position (astrometry) and intensity (photometry), and accurately estimate the planet's parameter uncertainties. It is indeed expected that the diverse set of postprocessing techniques would give rise to similar results. However, this is not necessarily the case as the signal-to-noise ratio $(\mathrm{S} / \mathrm{N})$ of a detected point source may vary significantly depending on the technique.

We present here a method implemented within a Bayesian framework: (1) to unify "source detection," i.e., deciding whether a certain signal is due to a source, and "source characterization," i.e., determining the parameters of the source, such as position, flux or intensity, into one single rigorous mathematical framework; (2) to enable an adequate hypothesis testing given the $\mathrm{S} / \mathrm{N}$ of the data; and (3) to enhance the detectability of faint signals from planets in the presence of noise and to optimize the characterization of the planet's position and flux. As a proof of concept we developed and implemented a module named PlanetEvidence that integrates the nested sampling technique (Multinest; Skilling 2004a, 2004b) with a postprocessing technique, the KLIP (Soummer et al. 2012). For the latter we use a python library for direct imaging of exoplanets and disks, PyKLIP (Wang et al. 2015) ${ }^{6}$, which uses an implementation of KLIP and KLIP-FM to perform PSF subtraction. This is a first step to recast such a postprocessing method into a fully Bayesian perspective. Furthermore our method can be applied equally to space-based and ground-based high-contrast images.

In Section 2. we give an overview of KLIP-FM, while in Section 3 we give an overview of Nested sampling and our implementation in PlanetEvidence. In Section 4, we present results and discussion for both $\beta$ Pictoris $b$ and synthetic data. Finally in Section 5 we present our conclusions.

\section{KLIP-FM: Detection and Forward Modeling-an Overview}

We extended an existing python library for direct imaging of exoplanets, PyKLIP (Wang et al. 2015) ${ }^{7}$, to the first stage of a fully Bayesian blind detection step.

We used the KLIP algorithm to do stellar PSF subtraction (Soummer et al. 2012). A companion algorithm to the KLIP method, the KLIP-FM allows for more accurate point-source detection using forward modeling (Pueyo 2016). KLIP-FM uses an input instrumental PSF at a given location and feeds it into the KLIP algorithm, thus resulting in a model instrumental PSF that can be corrected for biases due to PCA subtraction. This results in a model that has accounted for self- and over-subtraction of a

\footnotetext{
6 For details visit: https://pyklip.readthedocs.io/en/latest/.

7 For details visit: https://pyklip.readthedocs.io/en/latest/.
}
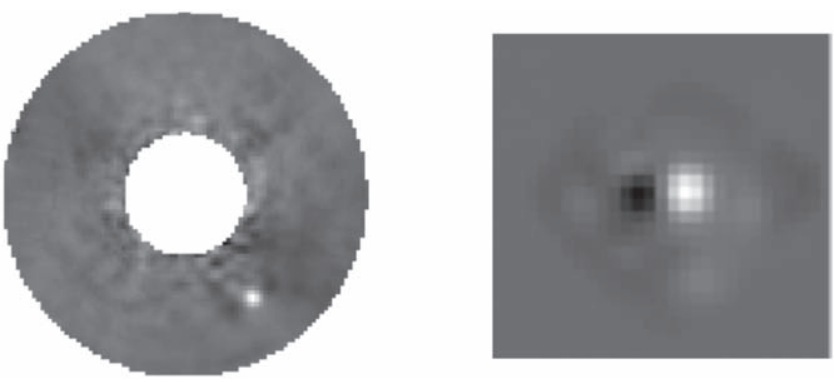

Figure 1. Left: KLIP-subtracted annulus from $\beta$ Pictoris $b$ image. Note the presence of the planet in the 5 o'clock position. Right: an enlarged instrumental PSF forward modeled in the location of $\beta$ Pictoris $b$.

planet signal in the guessed position of the planet. The initial instrumental PSF model is unique to the instrument used and is then propagated through KLIP. Here we make use of data from the Gemini Planet Imager (GPI) on the Gemini telescope (Macintosh et al. 2014). For the GPI data used, the instrumental PSF is constructed using satellite spots (manually-projected point sources) of known locations in the image.

The pyKLIP algorithm renders a KLIP-subtracted annulus that is plus and minus a user-prescribed number of pixels in the outward and inward radial directions from a guessed location of the planet. The guessed location of a planet is previously determined by eye from looking at the KLIP-subtracted image and looking for the presence of a planet. It also outputs another image, the post-KLIP instrumental PSF in the guessed location of the planet. This is then used as the model for the planet PSF in the image.

We explored one of two ways of extending pyKLIP to Bayesian detection and characterization: (i) starting with the coadded frames (sum of the set of temporal and wavelength frames) after subtracting the stellar PSF and (ii) starting with a joint analysis of all frames in time and wavelength. We present here approach (i) while approach (ii) will be completed in the future.

Following Soummer et al. (2012), we estimate the KL modes (up to some preset number) and subtract them from the image. Figure 1 shows a PSF-subtracted image of $\beta$ Pictoris (left-hand side). With a KLIP-subtracted image and the location of a potential planet in mind, we initialize our forward model in that location (in this case 30.1 pixels from the center and parallactic angle of $212^{\circ}$, measured counterclockwise from north). The fitting area, $\mathcal{F}$, is a $13 \times 13$ pixel box centered on the guessed location. For the detection step, we follow Wang et al. (2016): consider a set of parameters to minimize the assumed Gaussian-distributed residuals between the data and instrumental PSF model in given locations, while accounting for residual spatially-correlated background noise in the image. With $\mathrm{F}$ as the forward model of the instrumental PSF and D as the data, the aforementioned residuals over the fitting region $\mathcal{F}$ are defined as:

$$
R \equiv\left(D-\alpha F\left(x_{p}, y_{p}\right)\right)_{\mathcal{F}}
$$

The parameters introduced in this residual expression are spatial coordinates of the central location of the planet PSF $\left(x_{p}, y_{p}\right)$ as well as a flux scale parameter $\alpha$ to scale up or down the flux of the model to best match the data in $\mathcal{F}$.

The likelihood function introduced by Wang et al. (2016) also considers the correlated nature of residual noise in the KLIP-subtracted image. Such noise is accounted for in the Matérn covariance function $(C)$ with $\nu=3 / 2$ as motivated by Czekala et al. (2015). The covariance, $C$, between the $i_{\text {th }}$ and $j_{\text {th }}$ 
pixel is calculated as:

$$
C_{i j}=\sigma_{i} \sigma_{j}\left(1+\frac{\sqrt{3} r_{i j}}{\ell}\right) \exp \left(\frac{-\sqrt{3} r_{i j}}{\ell}\right)
$$

where $\ell$ is the correlation length scale, or the expected size of the residual correlated noise, and $r_{i j}$ is the distance between the $i^{\text {th }}$ and $j^{\text {th }}$ pixels in the image. It should be noted that $\ell \simeq \frac{\lambda}{D}$ (three pixels in our test case), but it is still treated as a parameter and allowed to vary. Also, $\sigma_{i}$ is the uncertainty associated with the $i_{\text {th }}$ pixel, computed by taking the standard deviation of pixel values in an annulus containing the $i_{\text {th }}$ pixel. The likelihood function is a Gaussian of the residuals with the aforementioned covariance matrix, giving the log likelihood:

$$
\ln \mathcal{L}=-\frac{1}{2}\left(R^{T} C^{-1} R+\ln (\operatorname{det}(C))+N_{\text {pix }} \ln (2 \pi)\right)
$$

where $\ln (\operatorname{det}(C))+N_{\text {pix }} \ln (2 \pi)$ is the normalization constant of the multivariate Gaussian.

\section{Nested Sampling: an Overview and Implementation in PlanetEvidence}

As opposed to getting the marginal distributions for each parameter using Markov-chain Monte Carlo sampling as done in Wang et al. (2016), we implement a nested sampling routine (introduced in Skilling 2004a, 2004b). Nested sampling was developed primarily to estimate the evidence (the average of the likelihood over the prior) for models being tested. As a byproduct it also provides the posterior distribution of the model parameters. Furthermore this sampling has a great overall speed of computation.

With the equation for the probability of a set of $n$ parameters, $\theta_{n}$, given data, $D$, as simply:

$$
P\left(\theta_{n} \mid D\right) \propto \mathcal{L}\left(D \mid \theta_{n}\right) \pi\left(\theta_{n}\right) .
$$

The expectation value of the likelihood with the priors $\pi\left(\theta_{n}\right)$ is this equation integrated over all of the parameters. This is called the evidence term, and is calculated by:

$$
Z=P(D)=\int \mathcal{L}\left(D \mid \theta_{n}\right) \pi\left(\theta_{n}\right) d \theta_{n} .
$$

Given that for many multiparameter models, this equation can become difficult or impossible to calculate, nested sampling is used to perform the calculation. In nested sampling, this multidimensional integral over parameter space is transformed into a onedimensional integral over probability space. The way this works is: $\mathrm{N}$ samples are made in prior space and the corresponding likelihood values are calculated. The lowest likelihood value is then banked (stored). It can therefore be said that there is an $\frac{N-1}{N}$ probability of getting that likelihood or greater when sampling from prior space. This is done iteratively, each time storing the lowest value likelihood value. Each iteration only samples from prior space that corresponds to likelihoods greater than the previously-stored lowest likelihood value, building up a function $L$ $(\lambda)$ (which is the probability of getting a likelihood of $\theta$ greater than $\lambda$ when sampling from the prior), the integral of which is calculated simply by summations. The equivalence between the integral over this probability space and that of the likelihood times the prior (Equation (5)) is explained in Skilling (2004a, 2004b). In addition, each parameter sample is assigned a corresponding weight to calculate marginal distributions for each parameter (for a detailed account see Skilling 2004a, 2004b). With this evidence term, we get an expectation value for the likelihood of the model given the data. It is worth noting that individual evidence values in its own right have no particular discriminatory meaning. It is only when the evidences for two competing models, $H_{0}$ (a null hypothesis) and $H_{1}$ (an alternative hypothesis), computed for the same data over the same fitting region are compared that one can draw conclusions.

For our purposes, we consider two models: one for the planet being present in the image, which we will call $H_{1}$, and another for just the noise present in the image, $H_{0}$ (null hypothesis). For $H_{1}$ model, we use the forward model with the same Matern covariance function to account for correlated noise. When we do sampling with this model, we are therefore looking for a point source, within a fitting region, that minimizes the residuals. For $H_{0}$ model, we assume there is no planet present in the fitting region, and we therefore set $\alpha=0$ and get the distributions for the remaining three parameters. Therefore, the likelihood is simply the likelihood of the data, with the Matern covariance to account for the correlated noise. The function for $H_{0} \log$ likelihood is therefore:

$$
\ln \mathcal{L}=-\frac{1}{2}\left(D^{T} C^{-1} D+\ln (\operatorname{det}(C))+N_{\text {pix }} \ln (2 \pi)\right) .
$$

For the nested sampling implementation, we use pyMultiNest (Buchner et al. 2014), a python wrapper for the multimodal nested sampling algorithm, called MultiNest (Feroz et al. 2009). This results in marginal distributions for all parameters, as well the evidence values $Z_{1}$ and $Z_{0}$ for the $H_{1}$ and $H_{0}$ hypothesis, respectively. Noting that the expressions for $Z_{1}$ and $Z_{0}$ are:

$$
Z_{1}=\int_{\theta=\ell, x, y, \alpha} \mathcal{L}(D \mid \theta) \pi(\theta) d \theta
$$

and

$$
Z_{0}=\int_{\theta=\ell, x, y} \mathcal{L}(D \mid \theta) \pi(\theta) d \theta .
$$

We can perform Bayesian model comparison to determine how much one model is favored over the other. In particular, the evidence for $H_{1}$ over $H_{0}$ is simply given by the ratio of $Z_{1}$ to $Z_{0}$, ie $B_{10}=Z_{1} / Z_{0}$. Note that pyMultinest gives values for $\ln Z$ rather than simply $Z$. The confidence for which one model can be favored over another from the evidence ratio can be determined using interpretation schemes such as, for example, the so-called "Jeffreys' scale" as tabulated in Trotta (2008) or the so-called "Harold-Jeffreys" interpretation (Jeffreys 1961).

\section{Results and Discussion}

\subsection{Results: Testing on $\beta$ Pictoris $b$}

We run our detection and characterization routine, $\mathrm{Pl}$ anetEvidence, on real direct imaging data, specifically the GPI data. We use the $J$-band coronagraph observations of $\beta$ Pictoris $\mathrm{b}$, an exoplanet with a model dependent mass of $10 M_{\mathrm{Jup}}$ at an orbital separation of 8-10 au (Lagrange et al. 2009, 2010). These observational data, along with those for several other targets, are available at the Gemini data release website. ${ }^{8}$ The $\beta$ Pictoris data come in the form of 19 time-series FITS data cubes, each containing 37 slices corresponding to exposures at different wavelengths ranging from 1.114-1.3497 microns.

\footnotetext{
8 https://www.gemini.edu/sciops/instruments/gpi/historical-documents/ public-data
} 


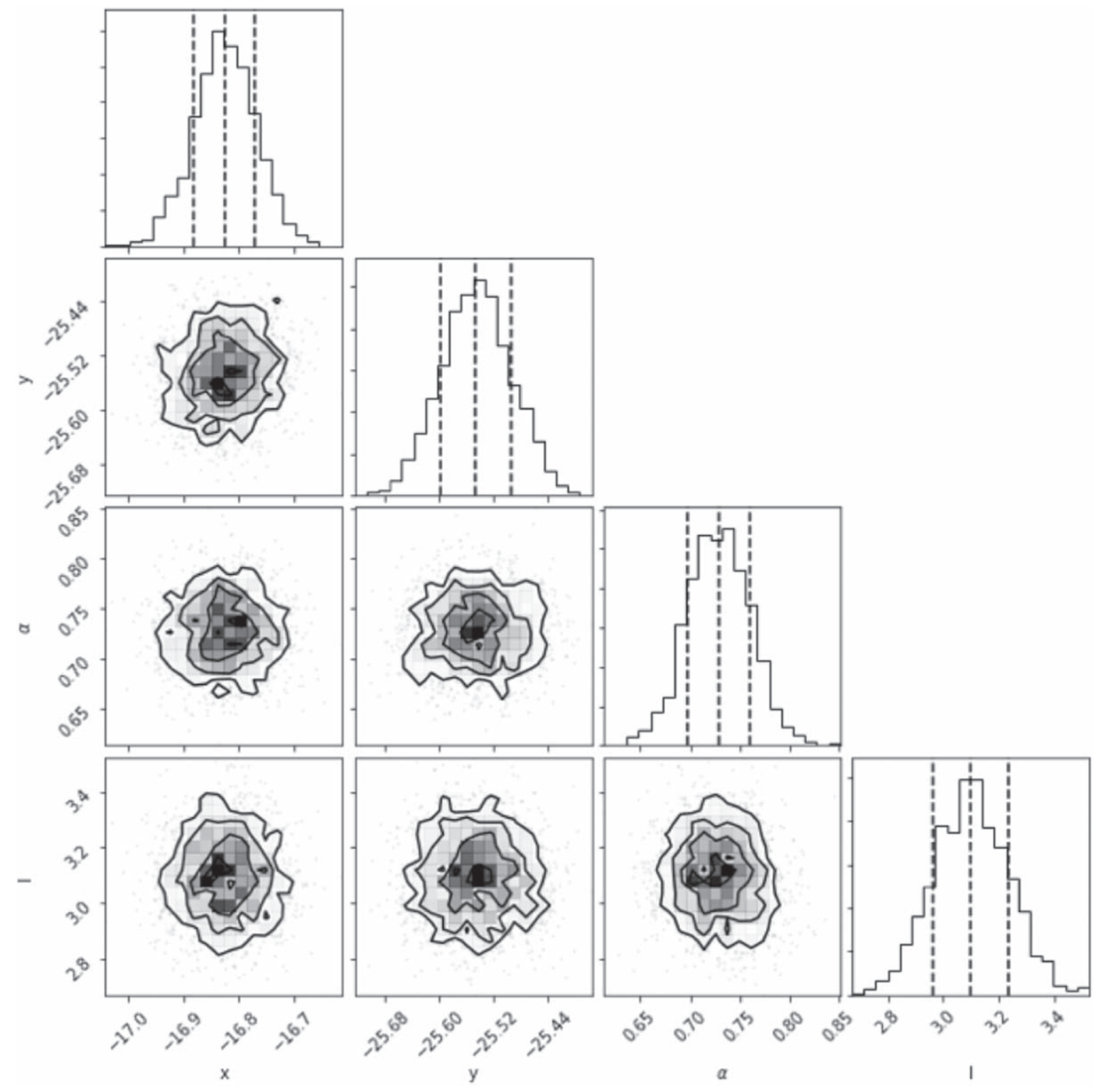

Figure 2. The posterior distributions of the four parameters of model $H_{1},(x, y, \alpha, \ell)$.

We consider the guessed location of the planet in the image at a radial separation of approximately 30.1 pixels from the center and at $212^{\circ}$ counterclockwise from the north of the image. We then use KLIP-FM to generate a forward model at that location. For the purposes of demonstration and consistency, we subtract $7 \mathrm{KL}$ modes from the data, following the examples used for $\beta$ Pictoris $\mathrm{b}$ in pyKLIP-FM. ${ }^{9}$ See Figure 1 for a KLIP-subtracted annulus of $\beta$ Pictoris $\mathrm{b}$ data, as well as the forward model generated for that location. We use MultiNest with the forward model to get the posterior distributions for each of the four parameters in the $H_{1}$ model.

The fitting area, $\mathcal{F}$, is a $13 \times 13$ pixel box centered on the guessed location. The priors for the central location of the planet's PSF is 1.5 pixels in both directions $(\mathrm{x}, \mathrm{y})$, while the priors for $\alpha$ and $\ell$ are uniform in $\log$ space with $\alpha$ between 0.016 and 1.58, and $\ell$ between 0.3 and 30 (as used in the example from pyKLIP forward modeling).

\footnotetext{
9 https://pyklip.readthedocs.io/en/latest/bka.html
}

$\mathrm{PlanetEvidence} \mathrm{renders} \mathrm{the} \mathrm{parameter} \mathrm{distributions} \mathrm{as}$ well as the overall evidence for both $H_{0}$ and $H_{1}$ hypothesis. The marginal distributions of the model parameters, the best-fit models, and the residuals are plotted in Figures 2-4.

Since $\beta$ Pictoris $\mathrm{b}$ is a bright planet at the guessed location in the image, we expect to recover parameter value with low uncertainties when fitting the forward model to the location of the planet in the image. This is clearly the case as shown in Figure 2. The distributions for the coordinates of the central position of the instrumental PSF have subpixel uncertainties (on the order of $+/-0.05$ pixels). This is consistent with (Wang et al. 2016; see Table 2 in the "Planet $\mathrm{X} / \mathrm{Y}$ uncertainty" column; the uncertainty on the planet position is 0.6 mas, which is $\approx 0.05$ pixels). Note that the accuracy of the estimated uncertainties of the position of beta Pictoris $\mathrm{b}$ are limited by unknowns such as beta Pictoris's position as well as astrometric calibration. The positional parameters are measured as displacement from the center of the image, and manually checking the spot where these parameters converge to in the image reveals it converges to the center of the location of $\beta$ Pictoris $\mathrm{b}$ (as determined by agreement with 


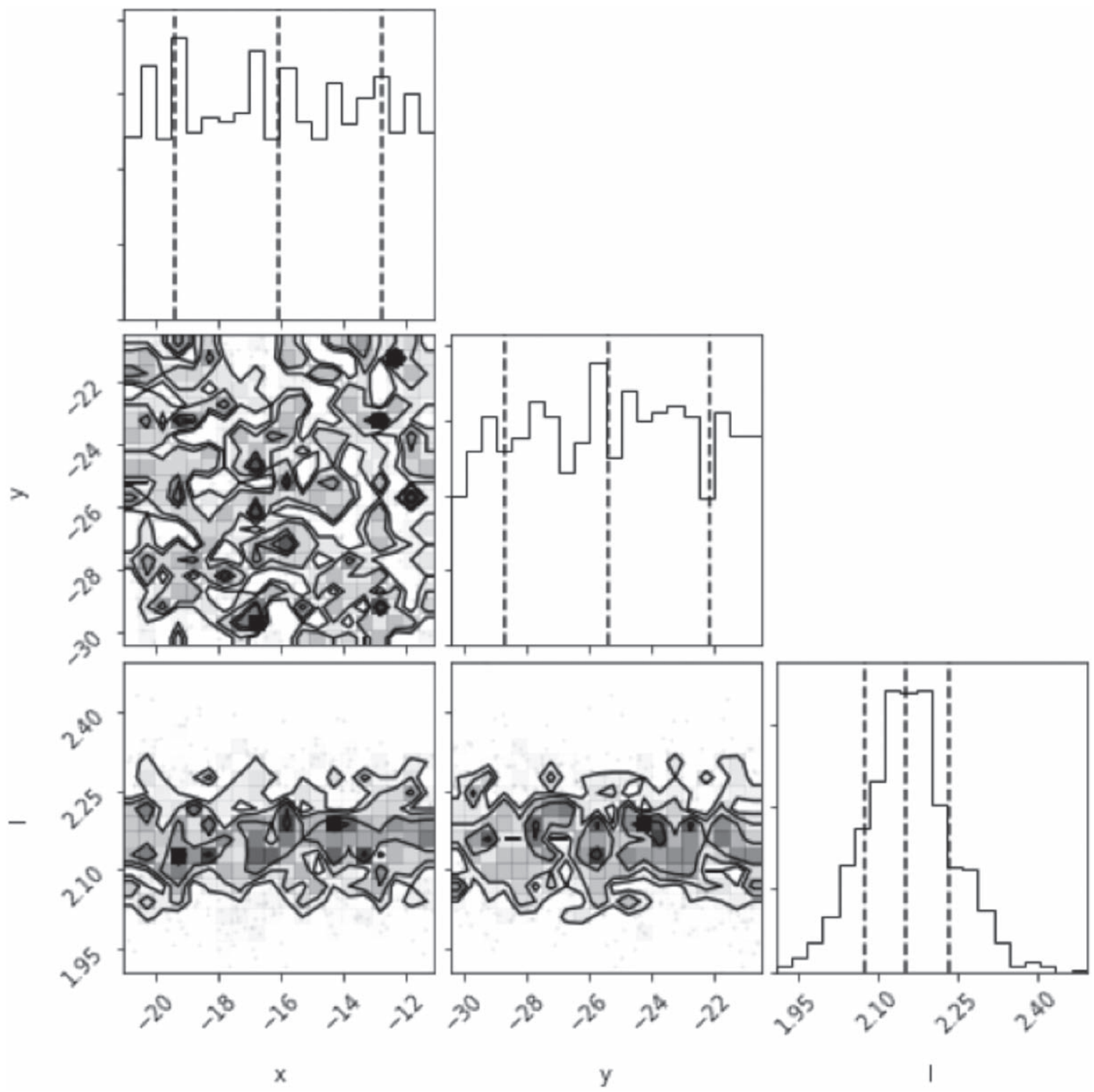

Figure 3. The posterior distributions of the three parameters of model $H_{0}$ (null hypothesis), $(x, y, \ell)$.
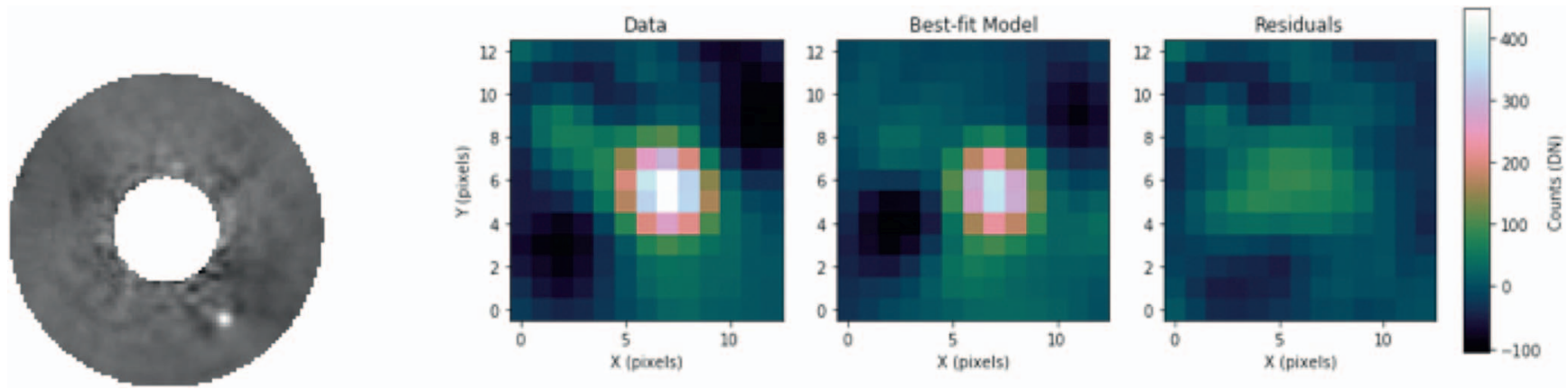

Figure 4. (Left) Annulus of KLIP-subtracted $\beta$ Pictoris b GPI data. (Right) Three-panel plot: (left) search region around $\beta$ Pictoris b, (center) the best-fit model for $H_{1}$ hypothesis, and (right) the residuals after subtracting the best-fit model. Residuals present in the right panel can be source flux residuals due to a potential underestimation of the source flux or residual background noise.

positional measurements from other publications; Wang et al. $2016)^{10}$. Also note that the $\alpha$ parameter converges to a value slightly less than 1 , as our forward model PSF was slightly

$\overline{10}$ https://pyklip.readthedocs.io/en/latest/bka.html brighter than the true brightness of $\beta$ Pictoris $b$. The forward model brightness is an initial guess of the planet flux, which is allowed to scale as a parameter in the fit (as we are just measuring how much to scale the input PSF to match the data; Wang et al. 2016). 
Table 1

$\beta$ Pictoris b: SNR and Evidence Ratios for Models $H_{1}$ (Planet Model) and the Null Hypothesis, $H_{0}$

\begin{tabular}{lcccc}
\hline \hline Target & $\mathrm{SNR}_{\mathrm{an}}$ & $\mathrm{SNR}_{\mathrm{bf}, \mathcal{F}}$ & $\left|\ln \left(B_{10}\right)\right|$ & Strength of Evidence \\
\hline$\beta$ Pictoris b & $11.4 \sigma$ & $11.5 \sigma$ & 175 & Very Strong \\
\hline
\end{tabular}

For $H_{0}$, the likelihood of just the data with the correlated residual noise is estimated along with the evidence for the null hypothesis. The posterior distributions of the three parameters of model $H_{0}$ are shown in Figure 3.

The logarithm of the evidence ratios, $\ln \left(B_{10}\right)=\ln \left(Z_{1} / Z_{2}\right) \approx$ 175 , very strong evidence in favor of the $H_{1}$ model. This is expected, as $\beta$ Pictoris $\mathrm{b}$ is a true, bright planetary signal in the image.

Note that we employ several methodologies to estimate the $\mathrm{S} / \mathrm{N}$ of a source. First, we calculate $\mathrm{SNR}_{\mathrm{bf}, \mathcal{F}}$, where the signal is considered the peak pixel of the best-fit model, and the noise is the standard deviation of the residuals within the fitting area $\mathcal{F}$. Note that this calculation of the $\mathrm{S} / \mathrm{N}$ is thus sensitive to both the accuracy of the fit (if the best-fit parameters are actually close to their true values) and the local residuals. For $\mathrm{S} / \mathrm{N}_{\mathrm{bf} \text {,an }}$ we use the peak of the best-fit model as the signal and the standard deviation of the masked annulus as the noise. Finally, $\mathrm{S} / \mathrm{N}_{\text {an }}$ is considered the brightest pixel at the injected location of the planet divided by the standard deviation of the planetmasked annulus (i.e., the SNR of the planet as seen in the image after going through the KLIP step), while $S / N_{\text {in,an }}$ is the input flux of the planet with noise estimated in the whole annulus.

For $\beta$ Pictoris $\mathrm{b}$, we calculate $\mathrm{S} / \mathrm{N}_{\mathrm{an}}=11.4 \sigma$, and the $\mathrm{S} / \mathrm{N}$ estimated from the residuals after subtracting the best-fit model within the fitting zone, $\mathrm{SNR}_{\mathrm{bf}, \mathcal{F}}=11.5 \sigma$. These $\mathrm{S} / \mathrm{Ns}$, along with the evidence ratio $\ln \left(B_{10}\right)=175$ are tabulated in Table 1 .

\subsection{Results: Testing on Synthetic Data}

To ascertain how dim a planet can be and still be detected using evidence ratios, we create controlled cases by injecting synthetic planets into different locations in the image of $\beta$ Pic $\mathrm{b}$, then running the detection and characterization routine.

Our goal is twofold: (1) to assess how faint a source can be at a given separation of the star and still be detected as a real source; and (2) to understand spatial variations of the noise in the annulus beyond the correlated noise described by the Matérn-Covariance matrix (in fact assessing the assumptions / limitations of the Matérn-Covariance matrix).

To inject a synthetic source we use pyklip.fakes module of pyKLIP. The fake sources are injected into the data before postprocessing (i.e., before running the KLIP step) at the correct positions so that after the data are aligned and rotated, the fake planet will be aligned. ${ }^{11}$.

For the sake of illustration we start by presenting detailed results for three locations in the image, with parallactic angle $p a=0 \mathrm{deg}$ (approximately located at 12 o'clock position on the image), $p a=270 \mathrm{deg}$ ( $\simeq 3$ o'clock), and $p a=90 \mathrm{deg} \quad(\simeq 9$ o'clock), followed by results for a larger sample of sources. All flux injection values are with respect to an initial flux of $5 \times 10^{-5}$ (this is an arbitrary value, approximately that of $\beta$ Pictoris b, prior to converting to contrast units for each

\footnotetext{
11 For details visit: https://pyklip.readthedocs.io/en/latest/contrast_curves. html/Injecting-fake-planets.
}

wavelength slice). We inject a planet in each of these locations with decreasing the $\mathrm{S} / \mathrm{N}$ by reducing the flux of the source down to $50 \%, 25 \%$, and $15 \%$ of the input flux, in order to demonstrate this detection and characterization routine for successively dimmer sources. To estimate the $\mathrm{S} / \mathrm{N}$, the noise is calculated after masking out both the synthetic planet and $\beta$ Pictoris $b$ (we mask a radius of five pixels centered at $\beta$ pic $b$ and another five pixels centered in the injected planet). The marginal distributions of the model parameters for these three positions at the progressively dimmer fluxes, the best-fit models, and the residuals are plotted in Figures 5-13. While the logarithm of the evidence ratios as function of the $S / N$ of the injected planets along with the strength of the evidence in favor of model $H_{1}$ are plotted on the left-hand side of Figure 14. Table 2 gives a summary of these results.

First, we consider $p a=0 \mathrm{deg}$ position. Results are shown in Figures 5-7 and tabulated in Table 2 . We start by injecting a planet at the $50 \%$ level, corresponding to an $\mathrm{SNR}_{\mathrm{an}}=7.5 \sigma$ and an $\mathrm{SNR}_{\mathrm{bf}, \mathcal{F}}=13.2 \sigma$. The posterior distributions of the source parameters plotted in Figure 5 show that the run for the $H_{1}$ model converges with the true location of the injected planet in the image. In contrast the posteriors distributions for the position parameters from the $H_{0}$ run are flat and have wide uncertainties (on the order of about \pm 3 pixels; see Figure 6). The logarithm of the evidence ratios, is $\approx 47$ strongly preferring $H_{1}$ over the null hypothesis. Next, in the same location, we inject a planet, this time at the $25 \%$ level, giving an $\mathrm{S} / \mathrm{N}_{\mathrm{an}}=4.3 \sigma$ and an $\mathrm{SNR}_{\mathrm{bf}, \mathcal{F}}=7.2 \sigma$. In this case the logarithm of the evidence ratios is 14 , still indicating a strong evidence in favor of the $H_{1}$ hypothesis. Finally, lowering the injected flux to $15 \%$, we recover an evidence ratio of $\approx 4.6$, corresponding to moderate-to-strong evidence in favor of $H_{1}$.

Next we inject a synthetic planet in a different location, now at $p a=270 \mathrm{deg}$ position. Results are shown in Figures 8-10 and tabulated in Table 2. Once again we start by injecting a planet at the $50 \%$ level, corresponding to $\mathrm{S} / \mathrm{N}_{\mathrm{an}}=5.5 \sigma$ and an $\mathrm{SNR}_{\mathrm{bf}, \mathcal{F}}=10.3 \sigma$. The logarithm of the evidence ratios is 42 , indicating a strong evidence for hypothesis $H_{1}$. When we reduce the injected flux to $25 \%, \mathrm{~S} / \mathrm{N}_{\mathrm{an}}=2.6 \sigma$ and an $\mathrm{SNR}_{\mathrm{bf}, \mathcal{F}}=5.3 \sigma$, and the logarithm of the evidence ratios is 11 , indicating still a strong evidence for $H_{1}$ model. Next, we reduce the injected planet flux to $15 \%$, with an $\mathrm{S} / \mathrm{N}_{\text {an }}=1.5 \sigma$ and an $\mathrm{SNR}_{\mathrm{bf}, \mathcal{F}}=2.9 \sigma$. The logarithm of the evidence ratios is 3.3 indicating moderate evidence at this location.

Finally we consider the position at $p a=90 \mathrm{deg}$. Results are shown in Figures 11-13 and tabulated in Table 2. Once again we start by injecting a planet with $\mathrm{S} / \mathrm{N}_{\mathrm{an}}=6.8 \sigma$ and an $\mathrm{SNR}_{\mathrm{bf}, \mathcal{F}}=8.0 \sigma$. The logarithm of the evidence ratios is 22, indicating a strong evidence for hypothesis $H_{1}$. When we reduce the injected flux to $25 \%, \mathrm{~S} / \mathrm{N}_{\mathrm{an}}=3.5 \sigma$ and an $\mathrm{SNR}_{\mathrm{bf}, \mathcal{F}}=2.4 \sigma$. The logarithm of the evidence ratios is 2.1, indicating moderate evidence for the $H_{1}$ model. Finally we inject a fainter planet in the same location with an $\mathrm{S} / \mathrm{N}_{\mathrm{an}}=2.2 \sigma$ and an $\mathrm{SNR}_{\mathrm{bf}, \mathcal{F}}=1.0 \sigma$. In this location, the $15 \%$ flux corresponds to an evidence ratio of 0.3 , indicating no evidence in favor of $H_{1}$. Thus, in this location at this dim flux level, the resulting low $\mathrm{S} / \mathrm{N}$ means the true planet cannot be distinguished from the surrounding background noise.

This simple exercise indicates that a planet with flux above a threshold of the order of $\mathrm{S} / \mathrm{N}_{\mathrm{an}} \sim 2.5 \sigma$ can, in principle, be detected relatively confidently using evidence ratios. From Figure 14 the resulting $\mathrm{S} / \mathrm{N}$ and evidence follow a similar trend 


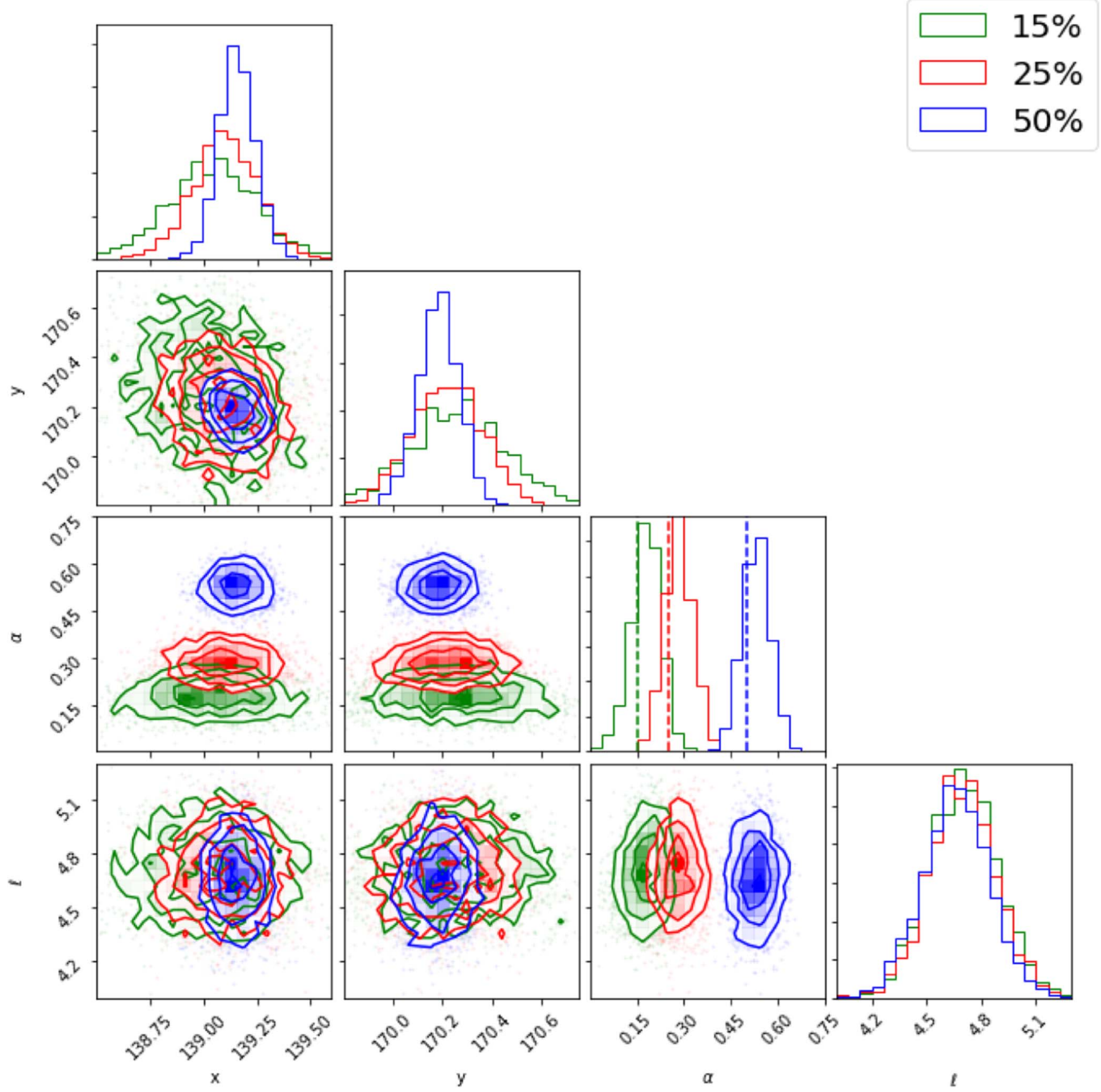

Figure 5. The posterior distributions of the four parameters of model $H_{1},(x, y, \alpha, \ell)$ for a planet injected at $p a=0$ deg (12 o'clock) position with $50 \%$ (blue), $25 \%$ (red), and $15 \%$ (green) of the forward-model flux $\left(5 \times 10^{-5}\right)$. Dashed lines correspond to the injected flux.

Table 2

The PSF Fractions, S/N, the Logarithm of the Evidence Ratios and the Strength of the Evidence in Favor of Model $H_{1}$ (Planet Model)

\begin{tabular}{|c|c|c|c|c|c|c|c|}
\hline Position = pa (deg) & $\mathrm{PSF}_{f}$ & $\left.\operatorname{SNR}_{\mathrm{bf}, \mathcal{F}}(\sigma)\right)$ & $\operatorname{SNR}_{\mathrm{bf}, \mathrm{an}}(\sigma)$ & $\operatorname{SNR}_{\text {in,an }}(\sigma)$ & $\mathrm{SNR}_{\mathrm{an}}(\sigma)$ & $\ln \left(B_{10}\right)$ & Strength \\
\hline 0 & 0.50 & 13.2 & 6.9 & 7.5 & 7.5 & 47 & Strong \\
\hline 90 & 0.50 & 8.0 & 5.2 & 7.4 & 6.8 & 22 & Strong \\
\hline 270 & 0.50 & 10.3 & 6.5 & 7.3 & 5.5 & 42 & Strong \\
\hline 0 & 0.25 & 7.2 & 3.6 & 3.8 & 4.3 & 14 & Strong \\
\hline 90 & 0.25 & 2.4 & 1.8 & 3.7 & 3.5 & 2.1 & Weak \\
\hline 270 & 0.25 & 5.3 & 3.3 & 3.7 & 2.6 & 11 & Strong \\
\hline 0 & 0.15 & 4.5 & 2.2 & 2.3 & 3.0 & 4.6 & Moderate \\
\hline 90 & 0.15 & 1.0 & 0.6 & 2.2 & 2.2 & -0.3 & Inconclusive \\
\hline 270 & 0.15 & 2.9 & 2.0 & 2.2 & 1.5 & 3.3 & Moderate \\
\hline
\end{tabular}



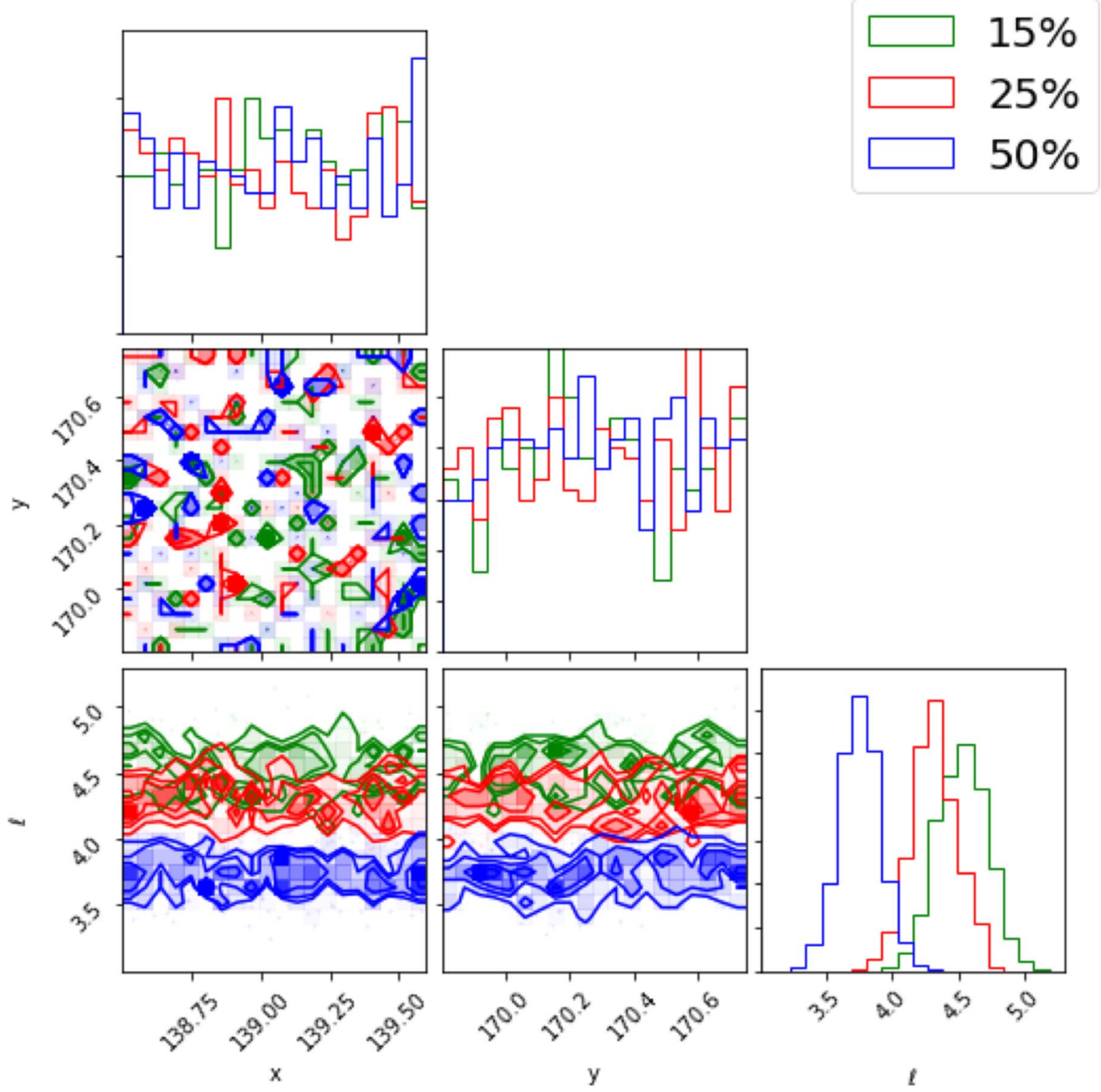

Figure 6. The posterior distributions of the three parameters of model $H_{0}$ (null hypothesis), $(x, y, \ell)$, for a planet injected at $p a=0$ deg (12 o'clock) position with $50 \%$ (blue), $25 \%$ (red), and $15 \%$ (green) of the forward model flux $\left(5 \times 10^{-5}\right)$.
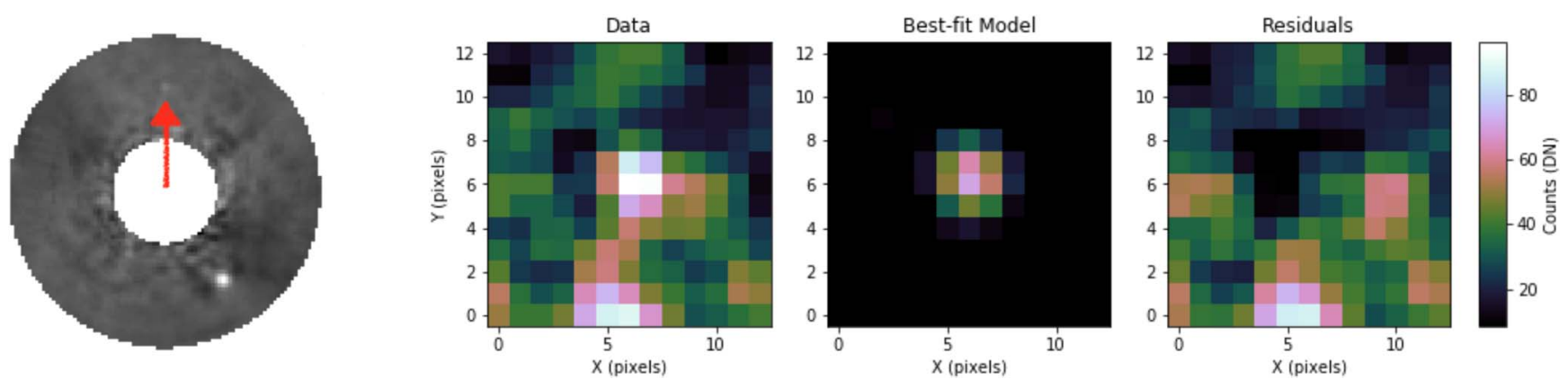

Figure 7. (Left) Annulus of KLIP-subtracted $\beta$ Pictoris b GPI data with a synthetic planet injected at $p a=0$ deg position with $15 \%$ of the forward model flux. (Right) Three-panel plot: (left) search region around the injected planet, (center) the best-fit model for $H_{1}$ hypothesis and (right) the residuals after subtracting the best-fit model. 


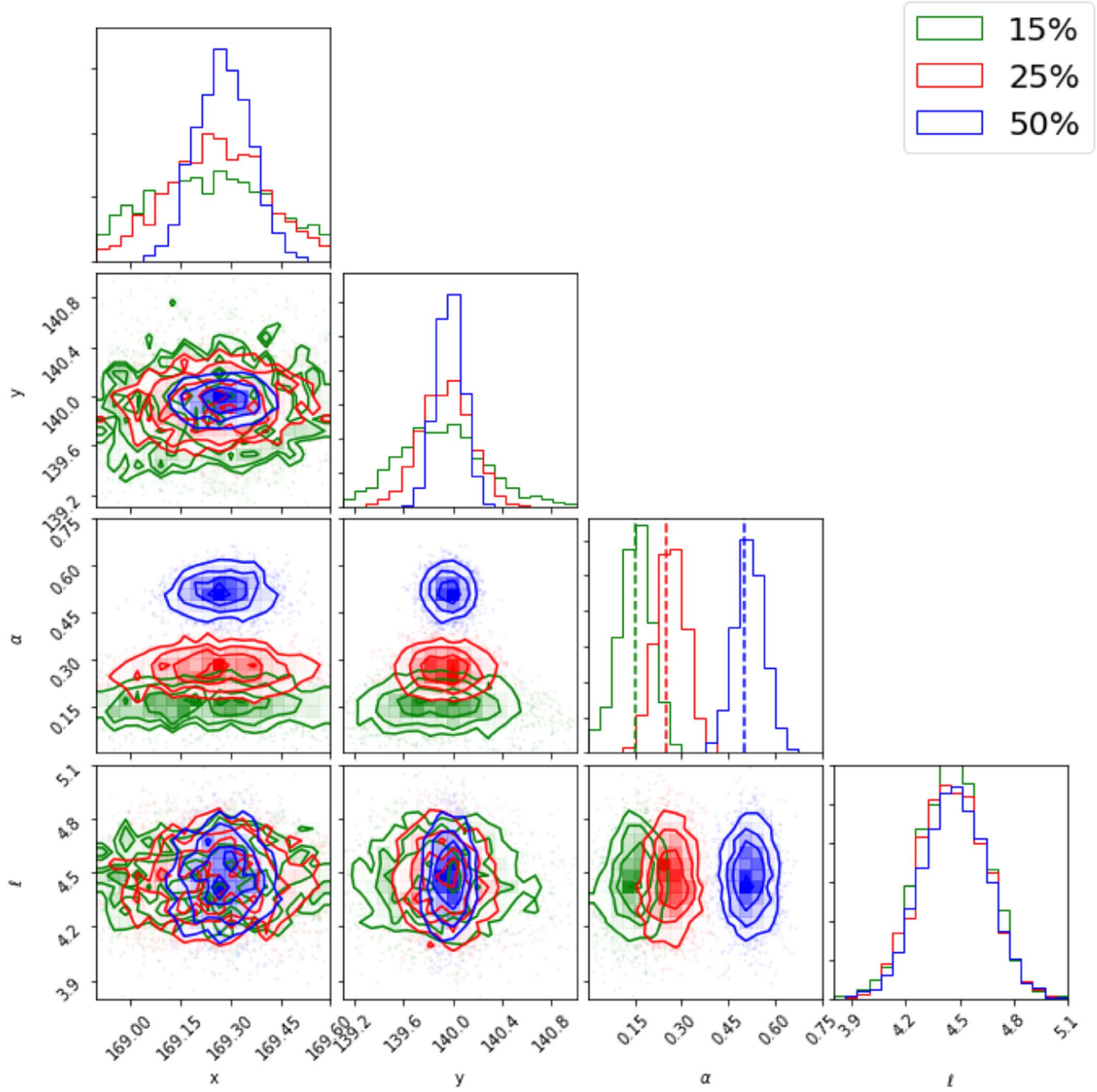

Figure 8. The posterior distributions of the four parameters of model $H_{1},(x, y, \alpha, \ell)$, for a planet injected at $p a=270$ deg (3 o'clock) position with $50 \%$ (blue), $25 \%$ (red), and $15 \%$ (green) of the forward-model flux. Dashed lines correspond to the injected flux.

for all cases. However the $p a=90 \mathrm{deg}$ ( 9 o'clock) position shows systematically lower $\mathrm{S} / \mathrm{N}$ for same fraction of the input flux injected and lower evidence ratios. The marginal distributions of the parameter $\alpha$ and the residual plots for the fainter injected planets plotted in Figures 5, 7, 8, 10, 11, and 13 show that the planet's flux is slightly overestimated for the $p a=0 \mathrm{deg}$ (12 o'clock) position, underestimated for the $p a=90 \mathrm{deg}$ (9 o'clock), and reasonably recovered, e.g., unbiased for the $p a=270 \mathrm{deg}$ ( 3 o'clock) location.

\subsection{Discussion of Primary Results}

Overestimating a planet's flux is possible if the injected planet falls partially or fully on top of speckle. Underestimation can occur when part of the planet's flux is subtracted when removing the residual speckles from the image in the KLIP step. This might happen if the chosen number of KL modes is insufficient to account for the noise characteristics and/or the KLIP procedure is insufficient (e.g., the reference frames chosen are inadequate) to properly separate both contributions, or if there is insufficient sky rotation. Mistakenly some of the planet's flux ends up contributing to the KLIP modes describing the residual speckles. The planet's flux is thus partially subtracted when the KLIP modes describing the speckles are subtracted from the image resulting in a underestimation of the true planet's flux rendered by the parameter $\alpha$. KLIP-FM should be compensating for these effects by 

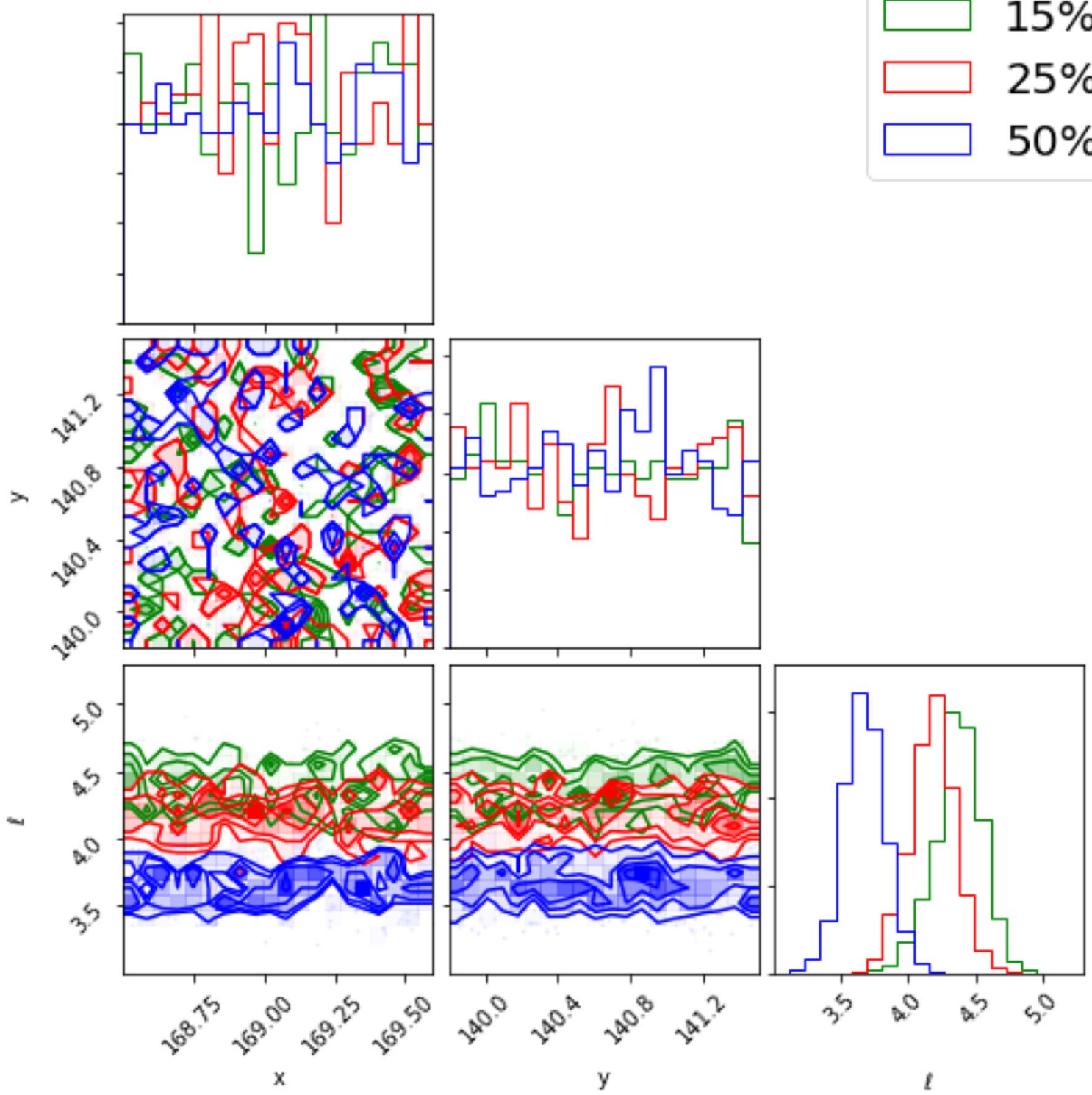

Figure 9. The posterior distributions of the three parameters of model $H_{0}$ (null hypothesis), $(x, y, \ell)$, for a planet injected at $p a=270$ deg $(3 \mathrm{o}$ 'clock) position with $50 \%$ (blue), $25 \%$ (red), and $15 \%$ (green) of the forward-model flux.
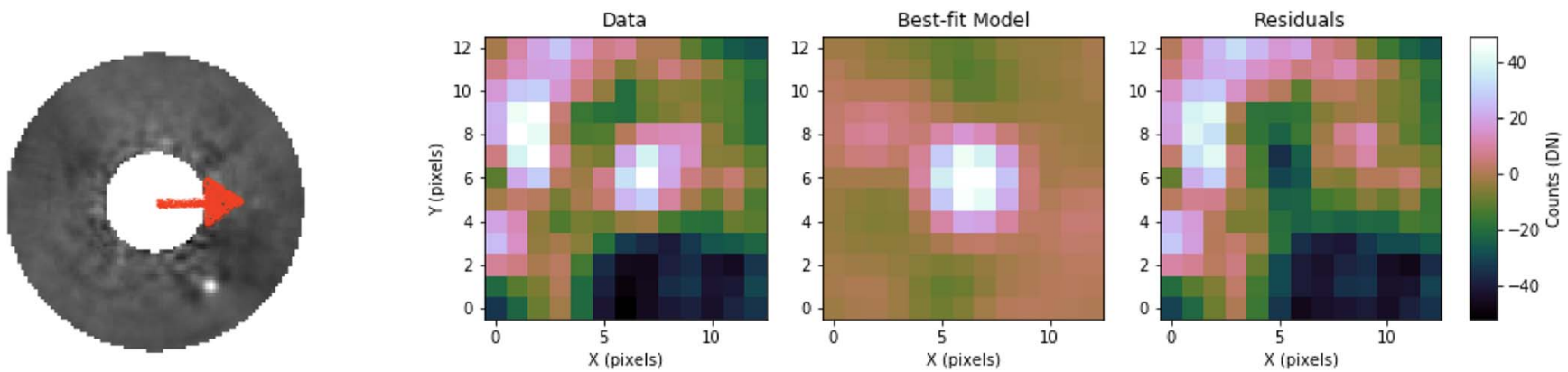

Figure 10. (Left) Annulus of KLIP-subtracted $\beta$ Pictoris b GPI data with a synthetic planet injected at $p a=270$ deg $(3$ o'clock) position with $15 \%$ of the forwardmodel flux. (Right) Three-panel plot: (left) search region around the injected planet, (center) the best-fit model for $H_{1}$ hypothesis and (right) the residuals after subtracting the best-fit model

accounting for the distortions of the original planet PSF due to the KLIP step. Hence this mild underestimation might indicate that the forward model does not completely account for the planet's PSF distortions at this position on the image. This or the log-uniform prior on the flux is dictating the fits as the
$\mathrm{S} / \mathrm{N}_{\mathrm{bf}, \text { an }}$ and $\mathrm{SNR}_{\mathrm{bf}, \mathcal{F}}$ are systematically low (and lower then the SNR on the other positions for the same planet's input flux).

We also note that the local $\mathrm{SNR}_{\mathrm{bf}, \mathcal{F}}$ is systematically higher then the $\mathrm{S} / \mathrm{N}_{\mathrm{bf}, \text { an }}$ for the three locations. The evidence values 


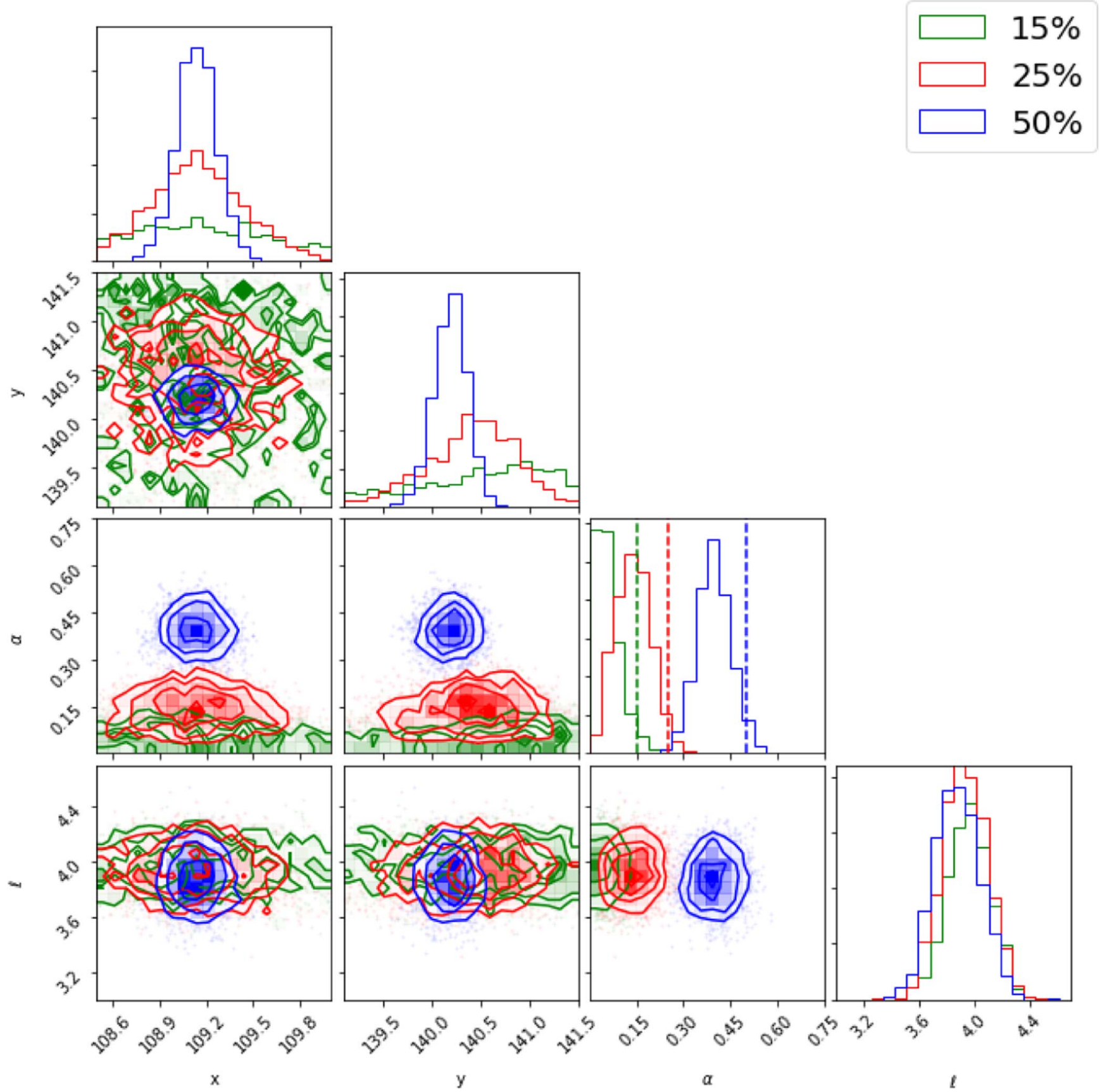

Figure 11. The posterior distributions of the four parameters of model $H_{1},(x, y, \alpha, \ell)$, for a planet injected at $p a=90$ deg (9 o'clock) position with $50 \%$ (blue), $25 \%$ (red), and $15 \%$ (green) of the forward-model flux. Dashed lines correspond to the injected flux.

and $\mathrm{S} / \mathrm{N}$ differences at each location for each injected flux show that the evidence for $H_{1}$ depends on the local noise estimated in the fitting region. It appears that the local fitted noise is systematically lower then the overall noise estimated in the annulus. This is due to the presence of a higher speckle residual noise in the inner boundary of the annulus as this region is not covered by the fitting regions around the selected locations of the injected planets.

\subsection{Synthetic Planet Injection Tests at Additional Locations}

To explore this further we inject a few sources at different locations in the annulus (at 1, 2, 3, 4, 6, 7, 8, 9, 11, 12 o'clock positions) considering 0.15 and 0.25 percentages of the FM that correspond to $\mathrm{S} / \mathrm{N}_{\mathrm{in} \text {,an }} 2.4 \sigma$ and $\mathrm{S} / \mathrm{N}_{\mathrm{in}, \text { an }} 3.7 \sigma$ respectively. The parallactic angle is measured in $30^{\circ}$ intervals counterclockwise, so $0^{\circ}$ is $12 \mathrm{o}^{\prime}$ clock, $30^{\circ}$ is $11 \mathrm{o}^{\prime}$ clock, $300^{\circ}$ is $2 \mathrm{o}^{\prime}$ clock, and $330^{\circ}$ is 1 o'clock.

On the right-hand side of Figure 14 we plot the $|\ln (B 10)|$ as function of the $\mathrm{S} / \mathrm{N}$ estimated in the local-fitting area $\mathrm{SNR}_{\mathrm{bf}, \mathcal{F}}$ for the several injected sources. We start by noting that the $\mathrm{SNR}_{\mathrm{bf}, \mathcal{F}}$ deviates from $\mathrm{S} / \mathrm{N}_{\mathrm{bf}, \text { an }}$ and $\mathrm{S} / \mathrm{N}_{\mathrm{an}}$. The differences with $\mathrm{S} / \mathrm{N}_{\mathrm{an}}$ can either indicate that the background noise is more complex then that described by a Matérn covariance matrix (the correlation length $\ell$ varies from 3 to 6 , the expected value is 3 ) and/or there is a over or underestimation of the 


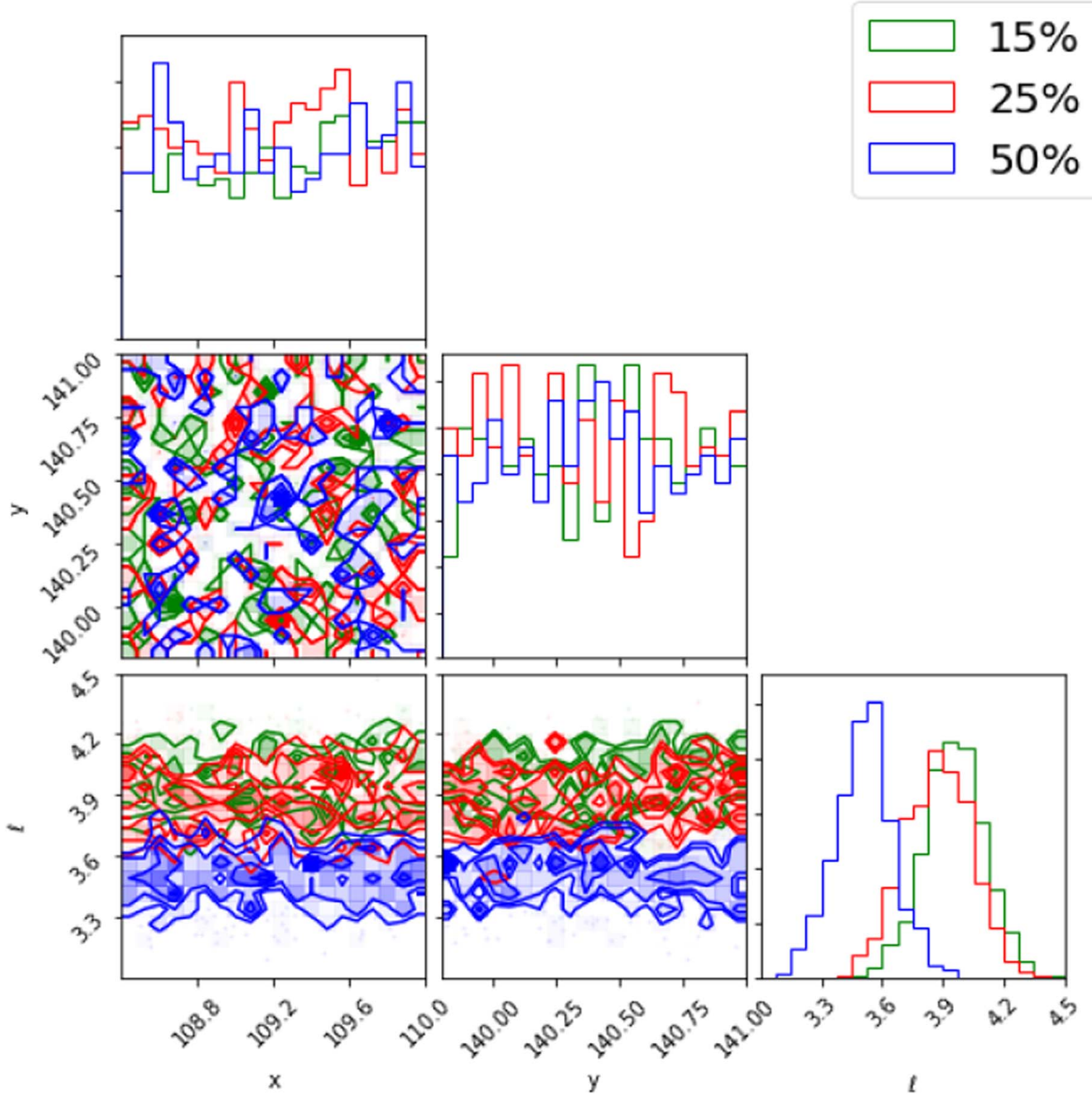

Figure 12. The posterior distributions of the three parameters of model $H_{0}$ (null hypothesis), $(x, y, \ell)$, for a planet injected at $p a=90 \mathrm{deg}(9 \mathrm{o}$ 'clock) position with $50 \%$ (blue), $25 \%$ (red), and $15 \%$ (green) of the forward-model flux.
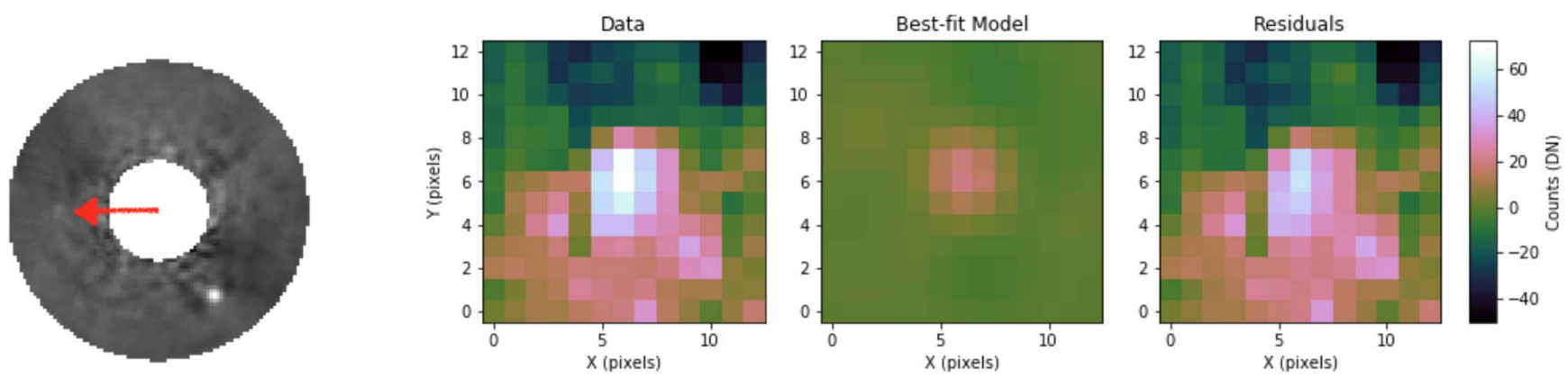

Figure 13. (Left) Annulus of KLIP-subtracted $\beta$ Pictoris b GPI data with a synthetic planet injected at $p a=90$ deg (9 o'clock) position with $15 \%$ of the forwardmodel flux. (Right) Three-panel plot: (left) search region around the injected planet, (center) the best-fit model for $H_{1}$ hypothesis and (right) the residuals after subtracting the best-fit model.

planet flux. This is so because $\mathrm{S} / \mathrm{N}_{\mathrm{an}}$ does not depend on the fitting procedure that renders the best-fit values of the flux parameter $\alpha$ and of the residual noise correlation length $\ell$ (along with their best posterior distributions).
For example, the 25 and 15 percentages of the planet's FM injected at $p a=90 \mathrm{deg}$ ( 9 o'clock) position exhibit lower $\mathrm{SNR}_{\mathrm{bf}, \mathcal{F}}$ then $\mathrm{SNR}_{\mathrm{an}}$. This is in agreement with the observed underestimation of its flux shown in Figure 11. While the flux 

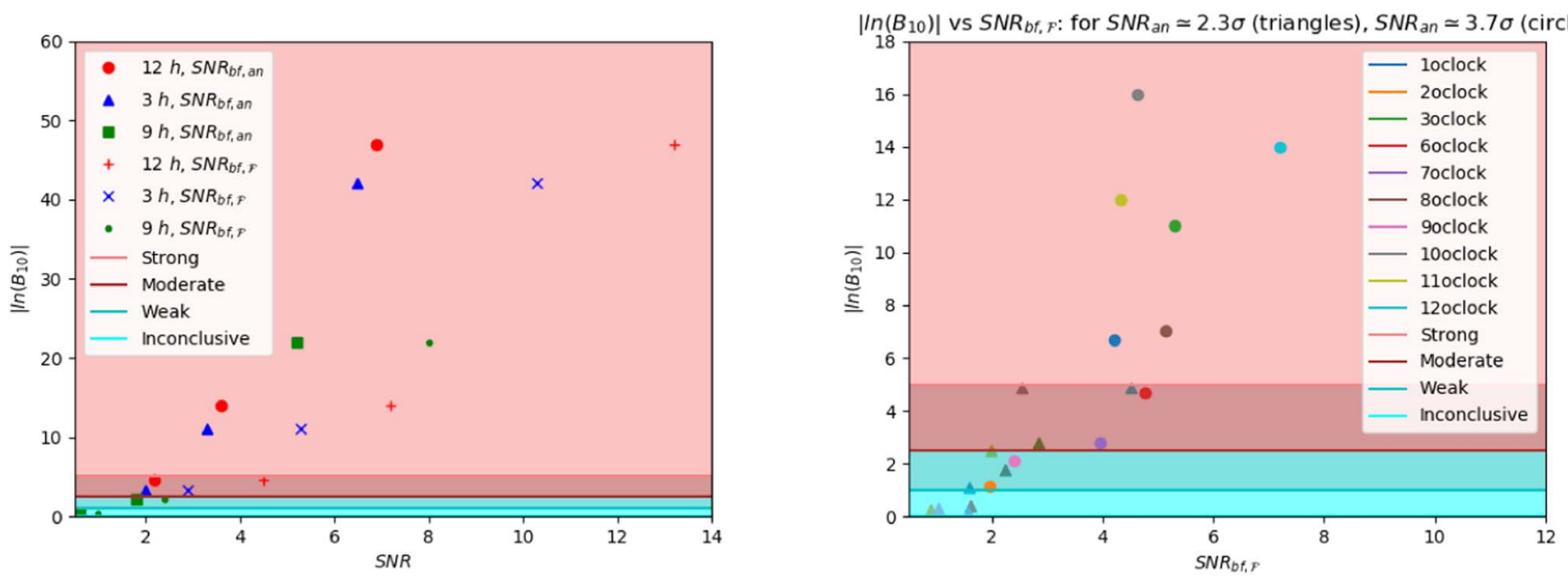

Figure 14. Evidence vs $\mathrm{S} / \mathrm{N}: \mathrm{S} / \mathrm{N}$ estimated in the annulus, $\mathrm{S} / \mathrm{N}_{\mathrm{an}}$, after masking $\beta$ Pictoris $\mathrm{b}$ and the injected planet; and $\mathrm{S} / \mathrm{N}$ estimated in the fitting area, $\mathrm{SNR} \mathrm{bf}_{\mathcal{F}} \mathcal{F}$ with noise estimated after subtracting the best-fit model from the fitting area. The horizontal lines represent the threshold values which are empirically set, and they occur for values of the $\operatorname{logarithm}$ of the Bayes factor of $|\ln B 10|=1.0,2.5$ and 5.0. Shaded areas represent the different levels of evidence above these thresholds according to convention in Trotta (2008).

Posterior Distributions for $\alpha$ (SNR 3.7 $\sigma$ )

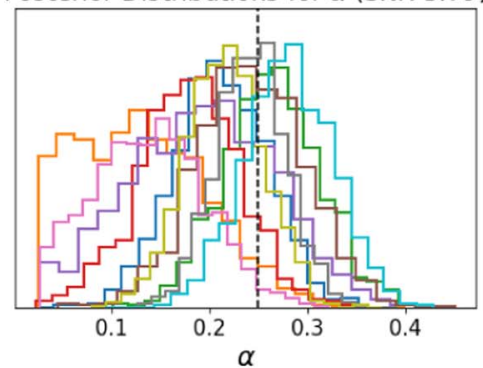

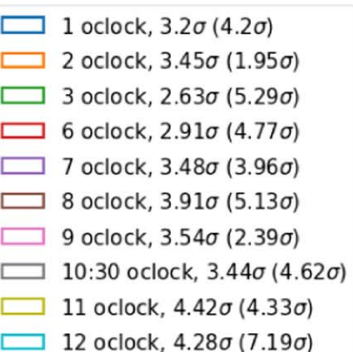

Posterior Distributions for $\ell$ (SNR $3.7 \sigma)$

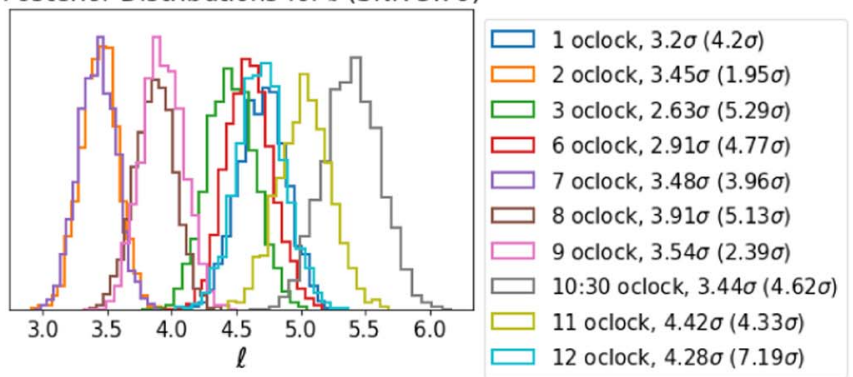

Figure 15. Left: posterior distributions of $\alpha$; right: posterior distributions of $\ell$ for all injected sources (ie for the several positions on the image) for $\mathrm{S} / \mathrm{N}_{\text {in,an }}$ $3.7 \sigma ; f_{\mathrm{FM}_{\mathrm{psf}}}=0.25$. First $\mathrm{S} / \mathrm{N}_{\mathrm{an}}$ is listed first, followed by $\mathrm{SNR}_{\mathrm{bf}, \mathcal{F}}$ in the parentheses. Dashed line corresponds to the injected flux.

\section{Posterior Distributions for $\alpha($ SNR $2.4 \sigma)$}

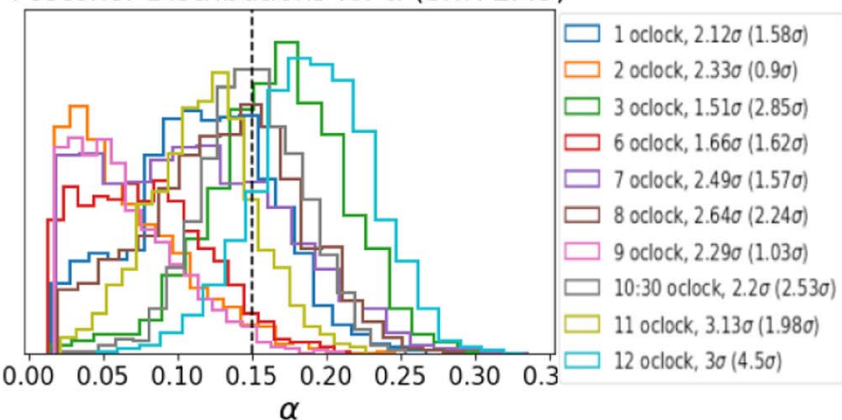

Posterior Distributions for $\ell$ (SNR 2.4 $\sigma$ )

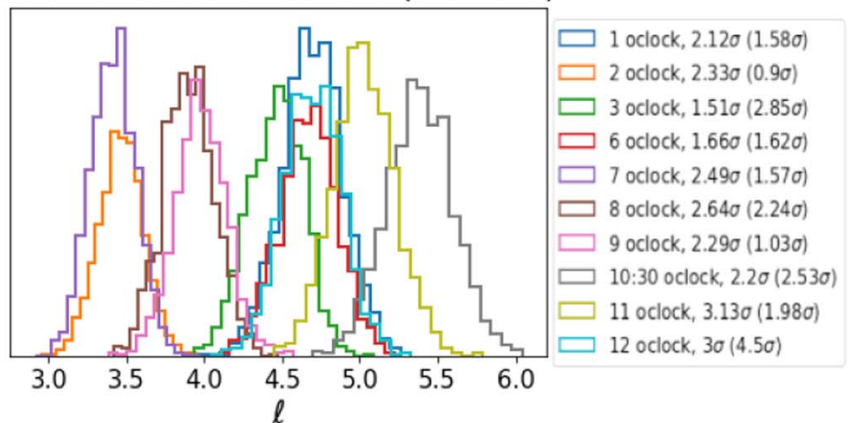

Figure 16. Left: posterior distributions of $\alpha$; right: posterior distributions of $\ell$ for all injected sources (ie for the several positions on the image) for $\mathrm{S} / \mathrm{N}_{\text {in,an }}$ $2.4 \sigma ; f_{\mathrm{FM}} \mathrm{psf}_{\mathrm{f}}=0.15$. The first $\mathrm{S} / \mathrm{N}_{\mathrm{an}}$ is listed first, followed by $\mathrm{SNR}_{\mathrm{bf}, \mathcal{F}}$ in the parentheses. Dashed line corresponds to the injected flux.

of the planet injected at $p a=270 \mathrm{deg}(3$ o'clock) position is reasonably recovered indicating that differences between these two $\mathrm{S} / \mathrm{N}$ estimators might be due to deficiencies in the noise characterization. Indeed there are larger scale correlations of noise in the image that we are ignoring because we are fitting a small local-fitting region.

Figures 15 and 16 show the posterior distributions of the source parameter $\alpha$ and the correlated residual noise (speckle) parameter $\ell$ for all positions in the image for $S / N_{a n} \simeq 3.7 \sigma$;
$f_{\mathrm{FM}_{\mathrm{psf}}}=0.25$ and $\mathrm{S} / \mathrm{N}_{\mathrm{an}} \simeq 2 \sigma ; f_{\mathrm{FM}_{\mathrm{psf}}}=0.15$. The marginal distributions of the parameter $\alpha$ indicate a clear flux underestimation by more than $1 \sigma$ for four of the ten locations in the image (clearly so in Figure 16), with skewed distributions toward lower values of $\alpha$. The posterior distributions are skewed to lower values when the $\mathrm{S} / \mathrm{N}$ is low. This seems to indicate that the prior distribution is influencing the posterior distribution (as mentioned above). Hence this underestimation could, in principle, also be due to 


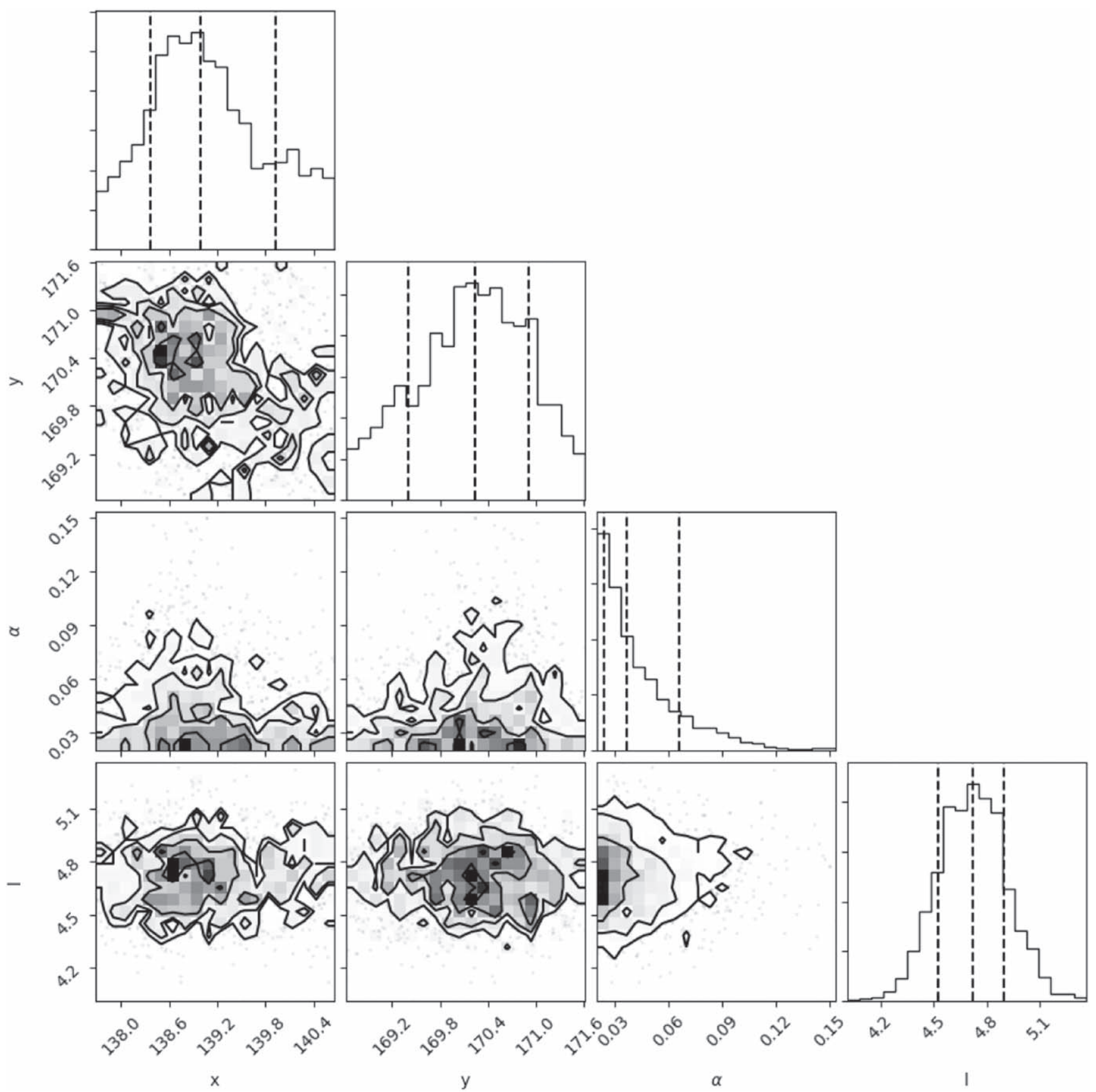

Figure 17. The posterior distributions of the four parameters of model $H_{1},(x, y, \alpha, \ell)$ for the runs of PlanetEvidence on noise, e.g., when no synthetic planet is injected into the data, at the $p a=0 \mathrm{deg}(12 \mathrm{o}$ 'clock) position in the image.

the choice of a log-uniform prior on the flux, rather than a uniform prior. (This will be further investigated and presented in a future publication.)

There is no clear correlation with the best-fit $\ell$ values. The peaks of the marginal distributions of the parameter $\ell$ fall in the range $3 \leqslant \ell \leqslant 6$. One would expect the distribution to peak around $\ell \sim 3$. This indicates the noise correlation length can be larger than expected. Explanation of the larger noise correlation length scale sizes may be subject of future work.

In this paper we consider Gaussian likelihoods and therefore we are not accounting for the potential non-Gaussianity of the real noise. This mismodeling of the statistics of the background noise can impact the estimation of the evidence. For example, in some cases, the fat tails of the non-Gaussian noise (Pairet et al. 2019) might give rise to a stronger evidence than it is in reality. The severity of this depends on how non-Gaussian the background noise is. In future work we will assess the resulting bias due to assuming a Gaussian noise. To this end we will construct non-Gaussian likelihoods following approaches described in Rocha et al. (2001, 2005).

Finally we tested this approach by running PlanetEvidence on "noise," when no synthetic planet is injected into the data. We consider the three $p a=0 \mathrm{deg}, p a=90 \mathrm{deg}$, and $p a=270 \mathrm{deg}$ positions in the image. Results are shown in 


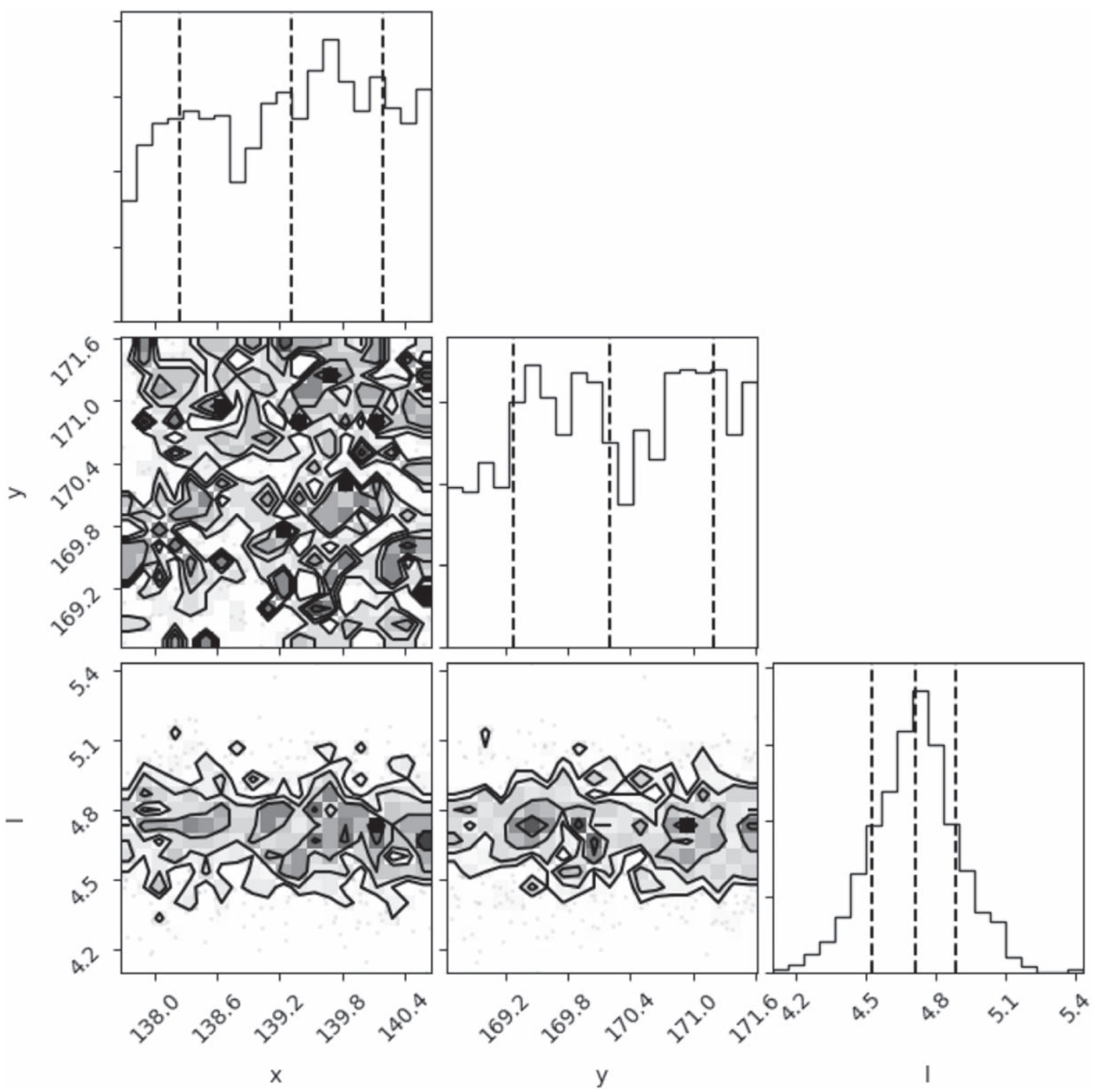

Figure 18. The posterior distributions of the three parameters of model $H_{0}$ (null hypothesis), $(x, y, \ell)$ for the runs of PlanetEvidence on noise, e.g., when no synthetic planet is injected into the data, at the $p a=0 \mathrm{deg}$ (12 o'clock) position in the image.

Figures 17-23, while the results are tabulated on Table 3 for all positions in the image.

We find that seven out of the ten cases considered hold $\mathrm{B} 10 \approx 0.3$ using Harold-Jeffreys scale (a scale to interpret the Bayes factor in model selection; Jeffreys 1961) interpretation for $B_{10}$ this indicates that Evidence supports the null hypothesis, $H_{0}$, while the remaining three cases are inconclusive.

In order to prove that the false-positive rate is low enough to confidently say they are planets a more thorough study is required. (In a future publication we will present such study by testing several different positions on background noise only simulations; note that here we use the observed data itself.) As it is, our results indicate that using evidence ratios we can detect "real" sources otherwise not seen by eye in the image.
Although KLIP-FM allows for accurate astrometry of a potential object, it does so given an initial guess of the pointsource location (for example, if detected by eye). Here we have shown that our method can relax this precondition, obviating the implementation of a blind detection step. This step will not use matched filters (as in the FMMF module in pyKLIP described in Ruffio et al. 2017), but rather incorporate all the information in the data model and likelihood and marginalize over the nuissance parameters. When implemented in the coadded data this step entails searching for planets in all pixels in the image and constructing a catalog of planets ordered according to the respective evidence value (following a similar procedure presented in Carvalho et al. 2009, 2012). 


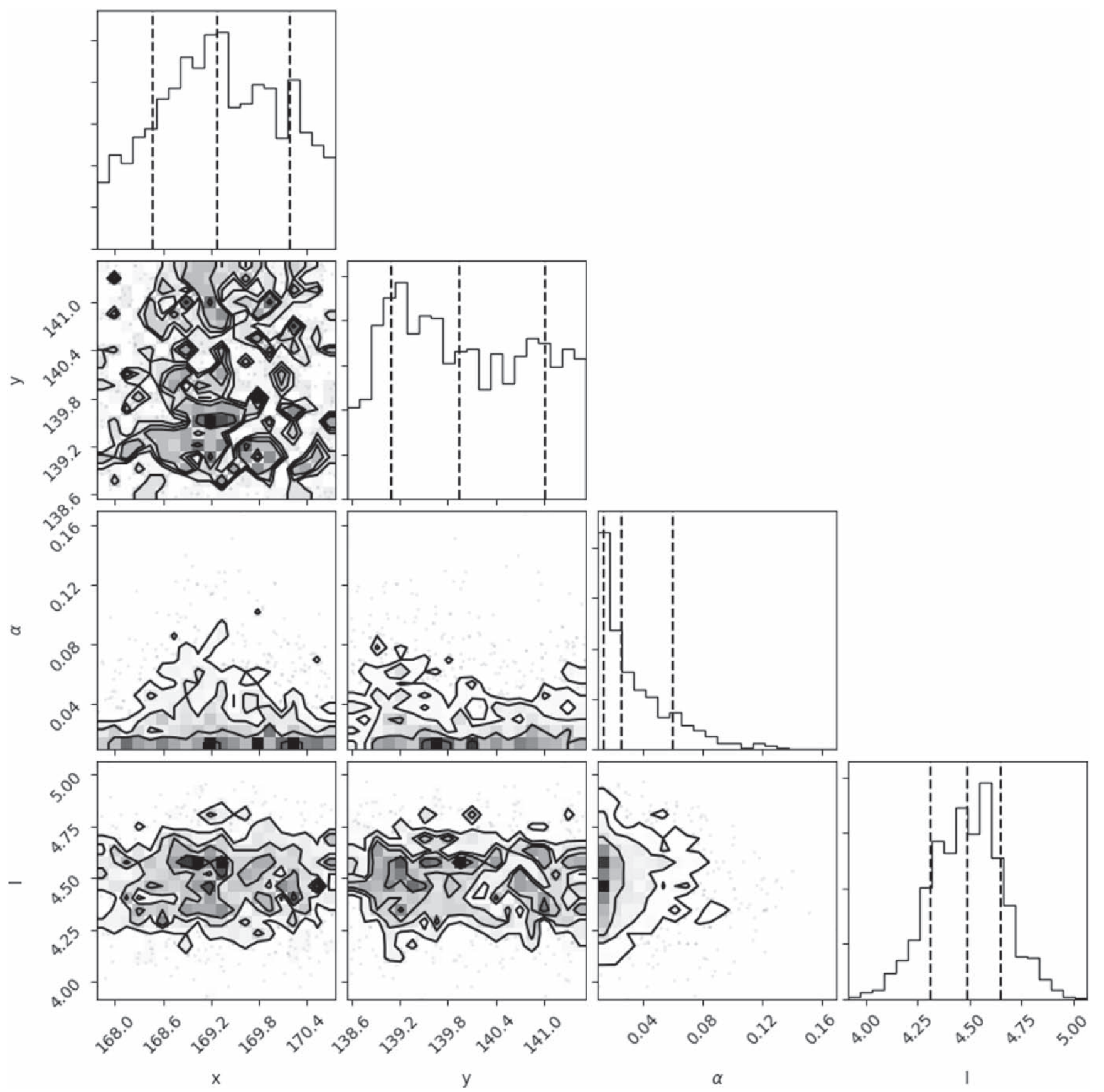

Figure 19. The posterior distributions of the four parameters of model $H_{1},(x, y, \alpha, \ell)$ for the runs of PlanetEvidence on noise, e.g., when no synthetic planet is injected into the data, at the $p a=270 \mathrm{deg}(3 \mathrm{o}$ 'clock) position in the image. 


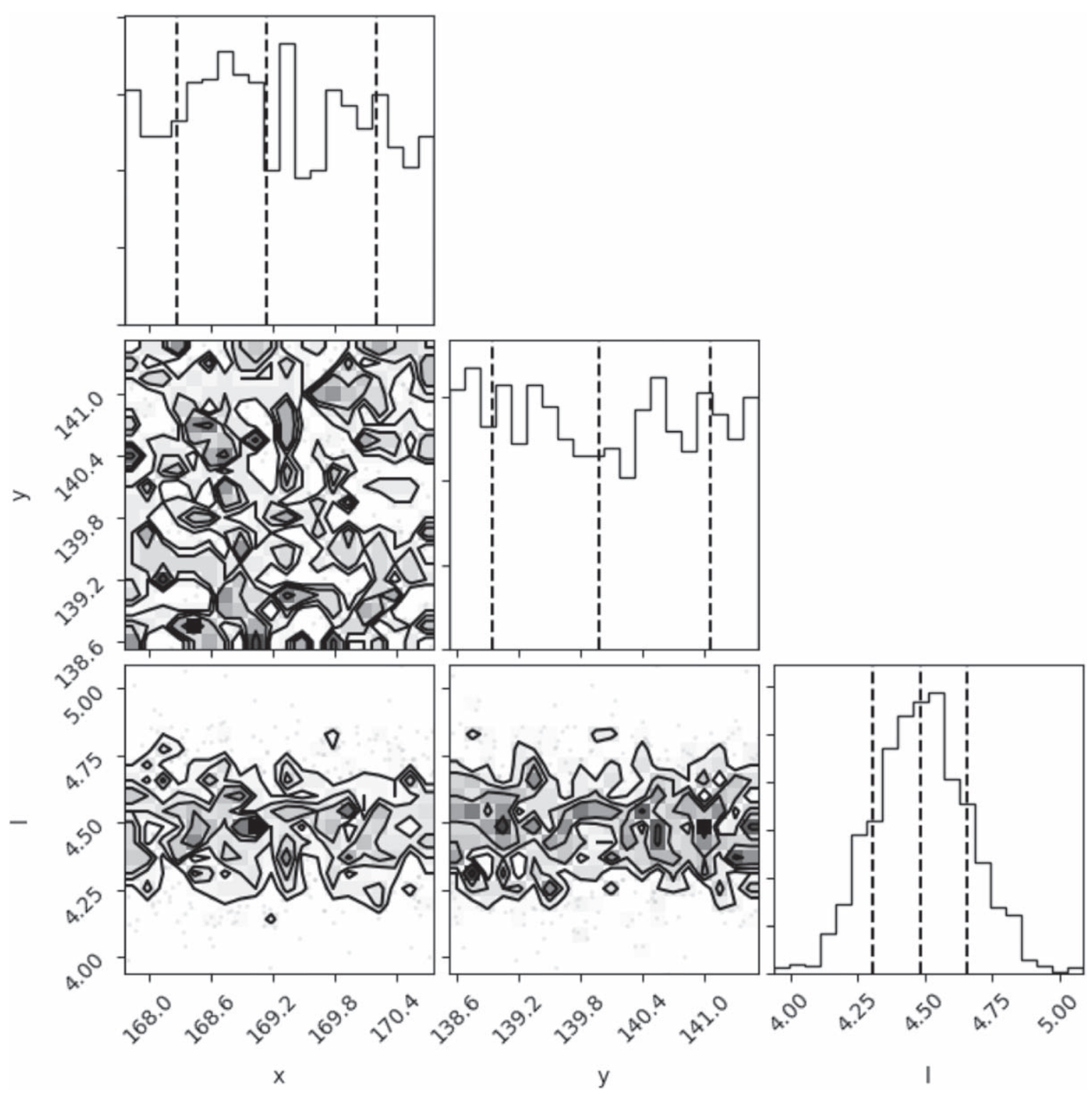

Figure 20. The posterior distributions of the three parameters of model $H_{0}$ (null hypothesis), $(x, y, \ell)$ for the runs of PlanetEvidence on noise, e.g., when no synthetic planet is injected into the data, at the $p a=270$ deg ( 3 o'clock) position in the image. 


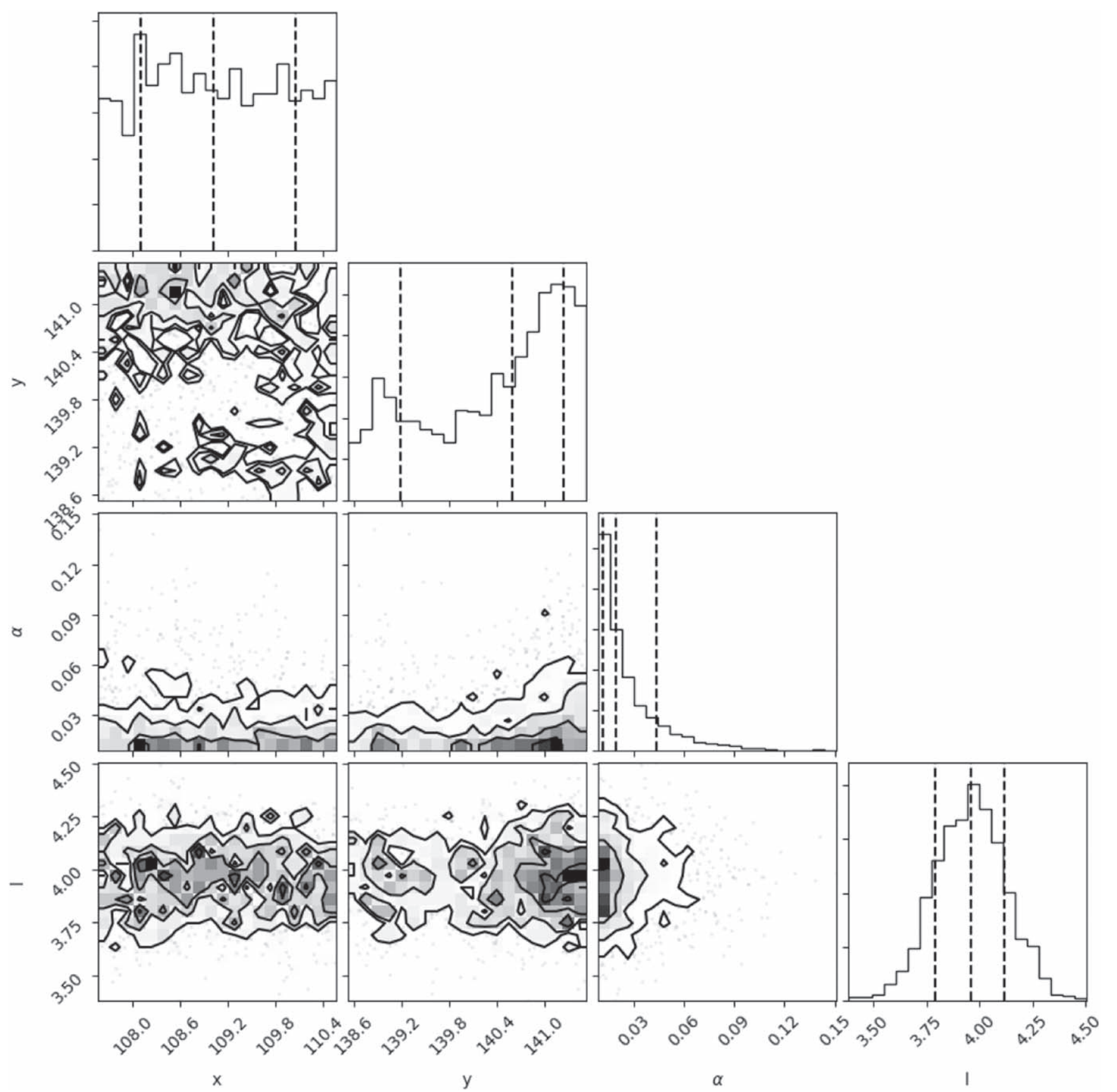

Figure 21. The posterior distributions of the four parameters of model $H_{1},(x, y, \alpha, \ell)$ for the runs of PlanetEvidence on noise, e.g., when no synthetic planet is injected into the data, at the $p a=90 \mathrm{deg}$ (9 o'clock) position in the image. 


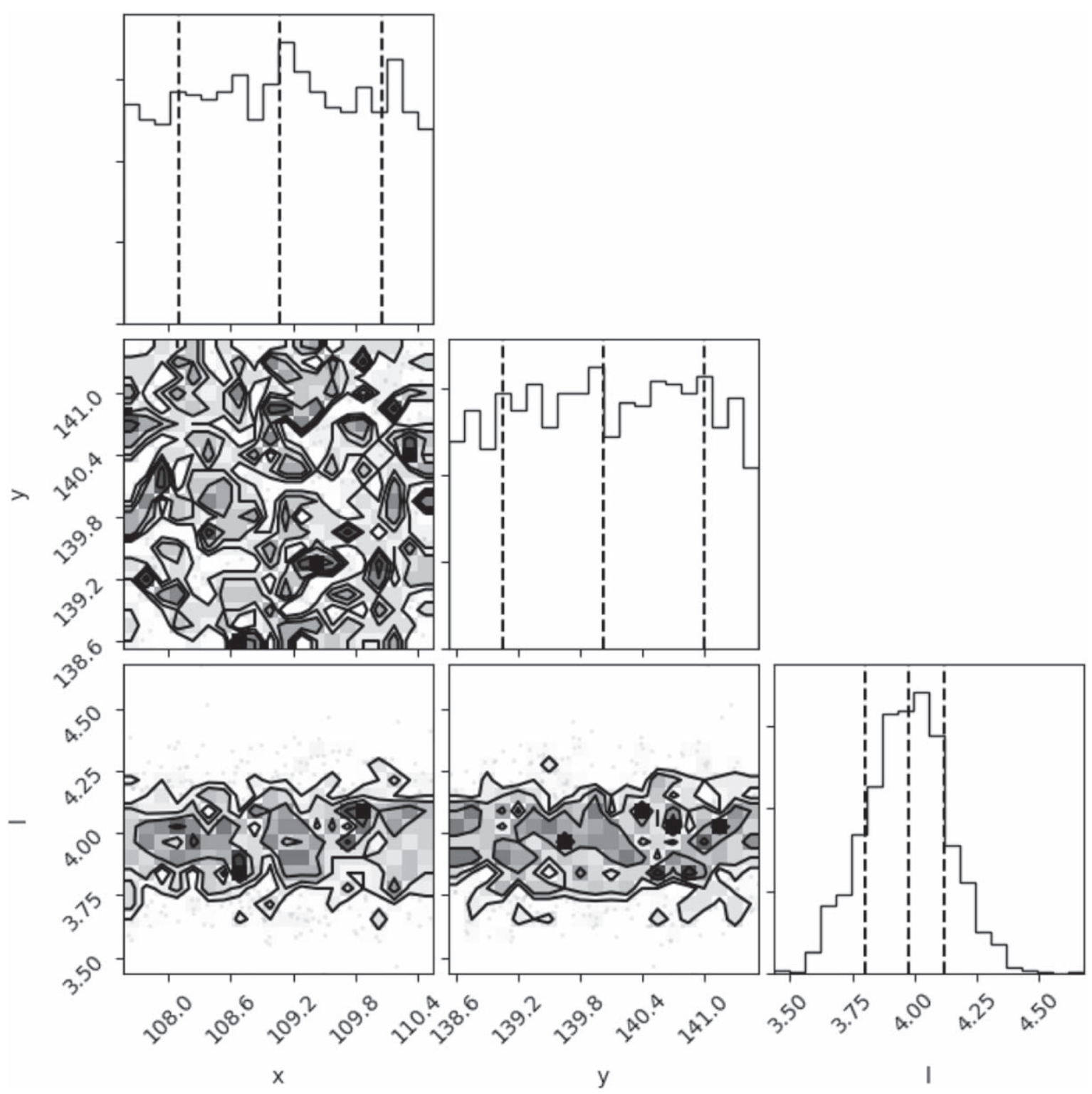

Figure 22. The posterior distributions of the three parameters of model $H_{0}$ (null hypothesis), $(x, y, \ell)$ for the runs of PlanetEvidence on noise ie when no synthetic planet is injected into the data, at $p a=90 \mathrm{deg}$ ( 9 o'clock) position in the image. 

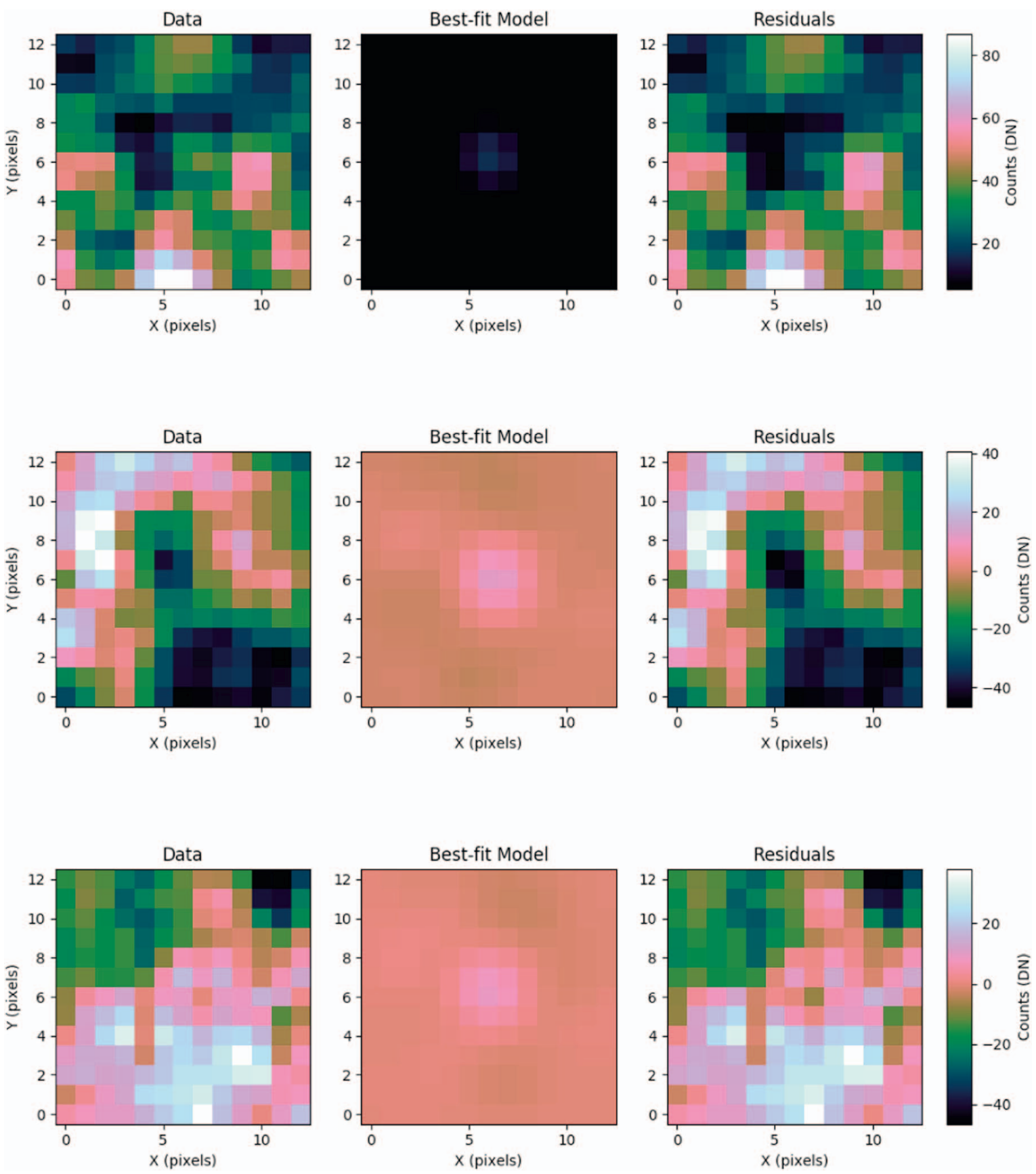

Figure 23. Three-panel plot: (left) search region around the chosen position on the image, (center) the best-fit model for $H_{1}$ hypothesis, and (right) the residuals after subtracting the best-fit model. (Top) for $p a=0 \mathrm{deg}$ (12 o'clock), (Middle) for $p a=270 \mathrm{deg}$ ( 3 o'clock), and (bottom) for $p a=90 \mathrm{deg}$ (9 o'clock). 
Table 3

The S/N, the Logarithm of the Evidence Ratios and the Strength of the Evidence in Favor of Model $H_{0}$ (Null Hypothesis) When No Synthetic Planet is Injected into the Data

\begin{tabular}{lccccc}
\hline \hline $\begin{array}{c}\text { Position }= \\
\text { pa }(\mathrm{deg})\end{array}$ & $\left.\mathrm{SNR}_{\mathrm{bf}, \mathcal{F}}(\sigma)\right)$ & $\mathrm{S} / \mathrm{N}_{\mathrm{an}}(\sigma)$ & $\ln \left(B_{10}\right)$ & $\mathrm{B} 10$ & Strength \\
\hline 0 & 1.1 & 1.7 & -1.2 & 0.3 & Weak \\
90 & 0.5 & 1.1 & -1.3 & 0.3 & Weak \\
270 & 0.6 & 0.4 & -0.9 & 0.4 & Inconclusive \\
30 & 0.574 & 2.061 & -1.4 & 0.3 & Weak \\
60 & 0.310 & -0.702 & 0.004 & 1.0 & Inconclusive \\
120 & 0.690 & 1.199 & -0.7 & 0.5 & Inconclusive \\
150 & 0.683 & 1.758 & -1.2 & 0.3 & Weak \\
180 & 0.594 & 0.945 & -1.1 & 0.3 & Weak \\
300 & 0.488 & 1.395 & -1.3 & 0.3 & Weak \\
330 & 0.553 & 1.045 & -1.3 & 0.3 & Weak \\
\hline
\end{tabular}

Note. A negative (positive) value for $\ln \left(B_{10}\right)$ indicates that the competing model is disfavored (supported) with respect to the null hypothesis $H_{0}$. Seven out of ten cases support the null hypothesis, $H_{0}$.

\section{Conclusions}

In this paper we introduced a methodology to determine whether a planet detected using KLIP-FM is a true point source rather than a residual background noise. This is achieved by constructing two models: $H_{1}$-a "planet is present" and $H_{0}$-null hypothesis ("no planet" present), and using nested sampling to compute the evidence for each model (i.e., we add an "evidence-based" metric to the postprocessing technique KLIP). We test this methodology by forward modeling on the location of $\beta$ Pictoris $\mathrm{b}$ and computing the corresponding evidence ratio for the $H_{1}$ and $H_{0}$ models. As expected, we get extremely strong evidence in favor for the planet being present rather than background noise. Next we test our approach on synthetic planets injected into the image. The evidence for the "planet present" hypothesis weakens as the $\mathrm{S} / \mathrm{N}$ of the injected source decreases. We have shown that dim sources, not necessarily seen by eye, can be detected and characterized as true point sources rather than background noise. To assess performance against false positives we tested our approach when no planet is injected. As expected, in most of the cases (seven out of ten), we get evidence in favor of the null hypothesis. The exercise presented here act as a proof of concept for a Bayesian-based algorithm for true blind detection; that is, not requiring an initial guess as to the location of the planet. As such, by initializing forward modeling at locations of interest (such as brighter points) across the KLIP-subtracted image, KLIP-FM may converge on a potential true source and the evidence that the detected source is more likely a true point source estimated with the PlanetEvidence module.

Our future implementation of a blind detection step in both coadded and uncoadded data differ from the FMMF module in pyKLIP described in (Ruffio et al. 2017, 2018). We will not use matched filters to boost the signal from the planet but rather incorporate the noise (both white and correlated noise) in the covariance matrix of the likelihood (assumed a multivariate Gaussian) and marginalize over the nuisance parameters. Our procedure follows the principles laid out in (Carvalho et al. 2009, 2012). Carvalho et al. (2009) have shown that maximizing the likelihood ratio between the two hypothesis, in the absence of the cross-term (see Equation 16 in Carvalho et al. 2012), with respect to the source amplitudes $A_{j}$, recovers the expression for the Matched Filter, MF. This means that the filtered field is merely the projection of the likelihood manifold on to the subspace of position parameters $X_{j}$. Hence they show that in the traditional approach to catalog making, in which one compares the maximum $\mathrm{S} / \mathrm{N}$ of the putative detections to some threshold, one is really performing a generalized likelihood ratio test. Furthermore, they lay out the foundations for a Bayesian-based generation of catalogs of point sources. In this Bayesian method, the threshold is a byproduct of the method rather than setup a priori (empirically), as is the case for frequentist-based methods.

PlanetEvidence is implemented in PyKLIP and is run in conjunction with KLIP-FM ${ }^{12}$

G.R. would like to acknowledge useful discussions with Jeff Jewell. The research presented here was carried out at the Jet Propulsion Laboratory, California Institute of Technology, under a contract with the National Aeronautics and Space Administration.

\section{ORCID iDs}

Graça Rocha (i) https://orcid.org/0000-0002-4150-8076 Tiffany Meshkat (1) https://orcid.org/0000-0001-6126-2467

Michael Bottom (다 https://orcid.org/0000-0003-1341-5531

Dimitri Mawet (i) https://orcid.org/0000-0002-8895-4735

Bertrand Mennesson (i) https://orcid.org/0000-00034205-4800

Jason Wang iㅏ https://orcid.org/0000-0003-0774-6502

\section{References}

Amara, A., \& Quanz, S. P. 2012, MNRAS, 427, 948

Bottom, M., Wallace, J. K., Bartos, R. D., Shelton, J. C., \& Serabyn, E. 2017, MNRAS, 464, 2937

Buchner, J., Georgakakis, A., Nandra, K., et al. 2014, A\&A, 564, A125

Cantalloube, F., Mouillet, D., Mugnier, L. M., et al. 2015, A\&A, 582, A89

Carvalho, P., Rocha, G., \& Hobson, M. P. 2009, MNRAS, 393, 681

Carvalho, P., Rocha, G., Hobson, M. P., \& Lasenby, A. 2012, MNRAS, 427, 1384

Czekala, I., Andrews, S. M., Mandel, K. S., Hogg, D. W., \& Green, G. M. 2015, ApJ, 812, 128

Feroz, F., Hobson, M. P., \& Bridges, M. 2009, MNRAS, 398, 1601

Jeffreys, H. 1961, The Theory of Probability (3rd edn.; Oxford: Oxford Univ. Press), 432

Lafrenière, D., Marois, C., Doyon, R., Nadeau, D., \& Artigau, É. 2007, ApJ, 660,770

Lagrange, A.-M., Kasper, M., Bocaletti, A., et al. 2009, A\&A, 506, 927

Lagrange, A.-M., Bonnefoy, M., Chauvin, G., et al. 2010, Sci, 329, 57

Macintosh, B., Graham, James R., Ingraham, Patrick, et al. 2014, PNAS, 111, 12661

Marois, C., Doyon, R., Racine, R., \& Nadeau, D. 2000, Proc. SPIE, 788, 788

Marois, C., Macintosh, B., Barman, T., et al. 2008, Sci, 322, 1348

Meshkat, T., Kenworthy, M. A., Quanz, S. P., \& Amara, A. 2014, ApJ, 780, 17

Mugnier, L. M., Cornia, A., Sauvage, J.-F., et al. 2009, JOSAA, 26, 1326

Nielsen, E. L., De Rosa, R. J., Macintosh, B., et al. 2019, AJ, 158, 13

Oppenheimer, B. R., \& Hinkley, S. 2009, ARA\&A, 47, 253

Pairet, B., Cantalloube, F., Gomez, G., et al. 2019, MNRAS, 487, 2

Pueyo, L. 2016, ApJ, 824, 117

Rocha, G., Magueijo, J., Hobson, M., \& Lasenby, A. 2001, PhRvD, 64, 063512

Rocha, G., Hobson, M. P., Smith, S., Ferreira, P., \& Challinor, A. 2005, MNRAS, 357, 1

Rosenthal, E. D., Gurwell, M. A., \& Ho, P. T. P. 1996, Natur, 384, 243

Ruffio, J.-B., Macintosh, B., Wang, Jason J., et al. 2017, ApJ, 842, 14

\footnotetext{
12 Visit https://pyklip.readthedocs.io/en/latest/ for PlanetEvidence release notes, examples and tutorials.
} 
Ruffio, J.-B., Mawet, D., Czekala, I., et al. 2018, AJ, 156, 196 Skilling, J. 2004a, in AIP Conf. Ser. (Melville, NY: AIP), 395

Skilling, J. 2004b, in AIP Conf. Ser. 735, Bayesian Inference and Maximum Entropy Methods in Science and Engineering. Am. Inst. Phys., New York, ed. R. Fischer, R. Preuss, \& U. V. Toussaint (Melville, NY: AIP), 395

Soummer, R., Pueyo, L., \& Larkin, J. 2012, ApJL, 755, L28
Trotta, R. 2008, ConPh, 49, 71

Vigan, A., Fontanive, C., Meyer, M., et al. 2020, A\&A, 651, A72

Wang, J. J., Ruffio, J.-B., De Rosa, R. J., Aguilar, J., Wolff, S. G., \& Pueyo, L. 2015, ascl.soft, Astrophysics Source Code Library, ascl:1506.001

Wang, J. J., Graham, J. R., Pueyo, L., et al. 2016, AJ, 152, 97

Ygouf, J. J., et al. 2016, AAS Meeting Abstracts, 227, 147.18 